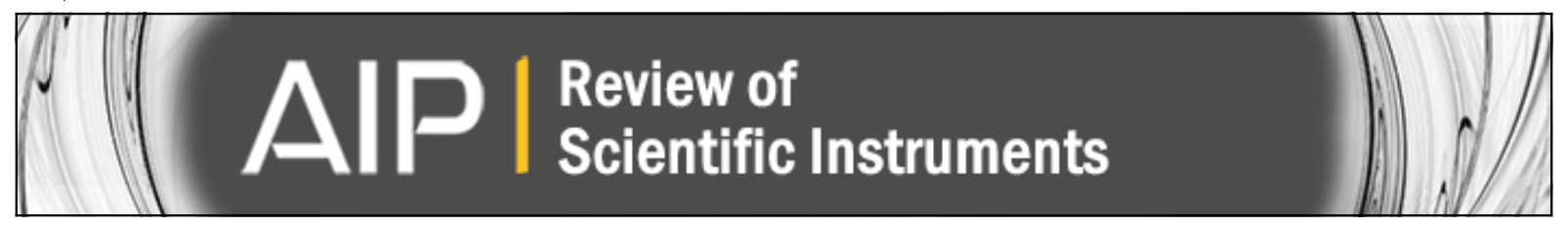

\title{
A high-field adiabatic fast passage ultracold neutron spin flipper for the UCNA experiment
}

A. T. Holley, L. J. Broussard, J. L. Davis, K. Hickerson, T. M. Ito, C.-Y. Liu, J. T. M. Lyles, M. Makela, R. R. Mammei, M. P. Mendenhall, C. L. Morris, R. Mortensen, R. W. Pattie, R. Rios, A. Saunders, and A. R. Young

Citation: Review of Scientific Instruments 83, 073505 (2012); doi: 10.1063/1.4732822

View online: http://dx.doi.org/10.1063/1.4732822

View Table of Contents: http://scitation.aip.org/content/aip/journal/rsi/83/7?ver=pdfcov

Published by the AIP Publishing

\section{AlP Re-register for Table of Content Alerts}

\section{Create a profile. \\ Sign up today!}




\title{
A high-field adiabatic fast passage ultracold neutron spin flipper for the UCNA experiment
}

\author{
A. T. Holley, ${ }^{1}$ L. J. Broussard, ${ }^{2}$ J. L. Davis, ${ }^{3}$ K. Hickerson, ${ }^{4}$ T. M. Ito, ${ }^{3}$ C.-Y. Liu, ${ }^{5}$ \\ J. T. M. Lyles, ${ }^{3}$ M. Makela, ${ }^{3}$ R. R. Mammei, ${ }^{6}$ M. P. Mendenhall, ${ }^{4}$ C. L. Morris, ${ }^{3}$ \\ R. Mortensen, ${ }^{3}$ R. W. Pattie, ${ }^{1}$ R. Rios, ${ }^{7}$ A. Saunders, ${ }^{3}$ and A. R. Young ${ }^{1}$ \\ ${ }^{1}$ Department of Physics, North Carolina State University, Raleigh, North Carolina 27695, USA \\ ${ }^{2}$ Department of Physics, Duke University, Durham, North Carolina 27708, USA \\ ${ }^{3}$ Los Alamos National Laboratory, Los Alamos, New Mexico 87545, USA \\ ${ }^{4}$ W. K. Kellogg Radiation Laboratory, California Institute of Technology, Pasadena, California 91125, USA \\ ${ }^{5}$ Department of Physics, Indiana University, Bloomington, Indiana 47405, USA \\ ${ }^{6}$ Department of Physics, Virginia Tech, Blacksburg, Virginia 24061, USA \\ ${ }^{7}$ Department of Physics, Idaho State University, Pocatello, Idaho 83209, USA
}

(Received 14 May 2012; accepted 18 June 2012; published online 18 July 2012)

\begin{abstract}
The UCNA collaboration is making a precision measurement of the $\beta$ asymmetry (A) in free neutron decay using polarized ultracold neutrons (UCN). A critical component of this experiment is an adiabatic fast passage neutron spin flipper capable of efficient operation in ambient magnetic fields on the order of $1 \mathrm{~T}$. The requirement that it operate in a high field necessitated the construction of a free neutron spin flipper based, for the first time, on a birdcage resonator. The design, construction, and initial testing of this spin flipper prior to its use in the first measurement of A with UCN during the 2007 run cycle of the Los Alamos Neutron Science Center's $800 \mathrm{MeV}$ proton accelerator is detailed. These studies determined the flipping efficiency of the device, averaged over the UCN spectrum present at the location of the spin flipper, to be $\bar{\epsilon}=0.9985$ (4). () 2012 American Institute of Physics. [http://dx.doi.org/10.1063/1.4732822]
\end{abstract}

\section{INTRODUCTION}

\section{A. Measuring the $\beta$ asymmetry with ultracold neutrons}

Free neutrons with a kinetic energy $T \lesssim 340 \mathrm{neV}$ $(v \lesssim 8 \mathrm{~m} / \mathrm{s})$ are called ultracold neutrons (UCN) and have energies well below the threshold at which their interaction with matter may be characterized by an effective constant potential (the volume-averaged Fermi potential)

$$
V_{F}=\frac{2 \pi \hbar^{2}}{m} n a,
$$

where $m$ is the neutron mass, $n$ is the number density of nuclei in the surrounding matter, and $a$ is the bound coherent nuclear scattering length. ${ }^{1,2}$ For many nuclei this material potential is positive and on the order of $100 \mathrm{neV}: \mathrm{Cu}$, for example, has $V_{F}^{\mathrm{Cu}} \approx 168 \mathrm{neV}$, while $V_{F}^{\mathrm{Be}} \approx 252 \mathrm{neV}$ and $V_{F}^{58} \mathrm{Ni} \approx 335 \mathrm{neV} .^{2}$ This means that many materials will reflect a large portion of the UCN spectrum for all angles of incidence. As a result, UCN guides and traps may be readily constructed using high potential materials directly, or by coating them onto a suitable substrate..$^{3-5}$ The small characteristic UCN energy also means that interaction of the nonzero neutron magnetic moment with a magnetic field is significant since a static field presents an accelerating potential of $-60 \mathrm{neV} / \mathrm{T}$ to neutrons whose spins are anti-aligned to the field and a retarding potential of $+60 \mathrm{neV} / \mathrm{T}$ to neutrons whose spins are aligned with the field. This allows, for example, a population of UCN to be essentially $100 \%$ polarized by passage through a $7 \mathrm{~T}$ magnetic field. Similarly, the gravitational potential has a significant effect on UCN energy since a
$1 \mathrm{~m}$ rise in the Earth's gravitational field corresponds to a potential energy change of about $100 \mathrm{neV}$. As a result, UCN trajectories are ballistic, causing them to undergo repeated collisions with confining surfaces, so that a population of UCN typically behaves like a dilute gas. (Current UCN sources provide densities in actual experiments which are generally less than $10 \mathrm{UCN} / \mathrm{cc}$.)

Some of the UCN attributes which derive from their very low characteristic energy, most notably their small probability (generally $10^{-4}$ to $10^{-5}$ ) for loss due to interaction with bulk matter of sufficiently high potential and their ability to be readily polarized, make UCN a very attractive system for high-precision measurements of neutron decay observables, used to gather experimental information about the charged weak current in the context of the standard model (SM) as well as to probe for physics beyond the SM. ${ }^{6}$ Such measurements typically require low backgrounds and often a high degree of polarization. ${ }^{7}$ In an effort to exploit the benefits of $\mathrm{UCN}$, the UCNA collaboration is for the first time using UCN to measure the $\beta$ asymmetry in polarized free neutron decay with a target precision $(<0.5 \%)$ which will ultimately provide a much-needed consistency check on similar measurements performed with cold neutron beams. ${ }^{8}$

The goal of a $\beta$ asymmetry measurement is to determine the relative probabilities of an electron emerging with its momentum parallel or anti-parallel to the neutron spin in polarized neutron decay $\left(\vec{n} \rightarrow p+e^{-}+\bar{v}_{e}\right)$. In practice, this can be accomplished by observing a population of polarized neutrons in a well-defined cylindrical volume, which contains a magnetic holding field parallel to the axis of the cylinder and of sufficient magnitude that all electron trajectories map to 
one end of the decay volume or the other. In this scheme, the difference in electron flux seen coming from the two ends of the decay volume provides the required relative probabilities. Since any asymmetry in the experimental configuration will distort the actual physics asymmetry, the ability to reverse the polarization of the neutron population is crucial because it allows constant differences in detector efficiencies to be removed to first order. ${ }^{8}$ This requirement necessitates a method of reversing neutron spins without changing the holding fields (which can potentially alter the relative detector efficiencies). In the case of an experiment using UCN, the ability to reverse the neutron spin in situ also enables a direct measurement of the equilibrium neutron polarization in each beta decay measurement cycle. ${ }^{9}$ Generally, the reversal of neutron polarization in such experiments is brought about by a radio frequency (rf) device which rotates the neutron spins through $180^{\circ}$, i.e., by an rf spin flipper.

\section{B. Figure of merit considerations for adiabatic fast passage spin flippers}

Using a combination of static and dynamic magnetic fields to manipulate populations of spins is the basis of nuclear magnetic resonance (NMR), and hinges on the wellknown fact that for an object with spin angular momentum $\boldsymbol{S}$ in a magnetic field $\boldsymbol{H}, \boldsymbol{S} \cdot \boldsymbol{H}$ is an adiabatic invariant of motion, i.e., spins tend to follow the local magnetic field as long as that field does not change too rapidly. ${ }^{10}$ The typical situation in NMR is to use a static holding field of magnitude $H_{0}$ to polarize a sample of spins in conjunction with a smaller perpendicular field of magnitude $H_{1}$ rotating around $H_{0}$ with angular frequency $\omega$ to manipulate them. The ability of the smaller $H_{1}$ field to affect the dynamics of the spins' evolution is due to a resonance effect, which may be understood intuitively by viewing a spin from a frame of reference which rotates around $H_{0}$ with angular frequency equal to the Larmor precession frequency $\omega_{\mathrm{L}}$ of the spin in $H_{0}\left(\omega_{\mathrm{L}}=-\gamma H_{0}\right.$, where $\gamma=-1.832 \times 10^{8} \mathrm{~T}^{-1} \mathrm{~s}^{-1}$ is the gyromagnetic ratio for a neutron). In that frame, the field $H_{0}$ must vanish since no precession of the spin due to $H_{0}$ will be observed, which means that if $H_{1}$ is also rotating with angular frequency $\omega_{\mathrm{L}}$ it will appear static and the spin will precess solely around it. In the lab frame the spin will be reoriented due to both its precession around $H_{1}$ and the rotation of $H_{1}$ itself. $H_{1}$ will continue to dominate the spins' evolution in the rotating frame, and thus have the ability to reorient the spin in the lab frame, as long as $\omega-\omega_{\mathrm{L}}$ is not too large, i.e., as long as the movement of $H_{1}$ in the rotating frame does not violate adiabaticity.

In 1970 V. Luschikov suggested that a technique from NMR called adiabatic fast passage (AFP), where the rotation frequency of the $H_{1}$ field is swept through the resonance at $\omega$ $=\omega_{\mathrm{L}}$ in order to produce a $\pi$-flip in a sample of spins, might be utilized to produce in-beam spin flipping over a wide range of velocities and thus an effective UCN spin flipper. ${ }^{11}$ Instead of changing the $H_{1}$ rotation frequency, however, the idea was to give the holding field $B_{0}$ a monotonic gradient. Then, as a neutron travels through the region of non-zero $B_{1}$, its Larmor precession frequency due to $B_{0}$ is always either increasing or decreasing (depending on the direction of travel). If the rotation frequency $\omega$ of $B_{1}$ is chosen to be equal to $\omega_{\mathrm{L}}\left(z_{r}\right)$ at a point $z_{r}$ inside the region of non-zero $B_{1}$, it turns out that a spin which passes completely through the rotating-field region, regardless of the direction of travel, will undergo a $\pi$-flip irrespective of its actual trajectory as long as adiabaticity is maintained; this corresponds, for example, to a neutron passing through the enlarged region in Fig. 1. If, on the other hand, the spin does not pass completely through the non-zero $B_{1}$ region, i.e., if it scatters back out the way it came, then its spin direction will remain unchanged.

Abragam ${ }^{10}$ demonstrates this effect by considering a region in empty space with a total magnetic field given in the lab frame (frame I) by

$$
\boldsymbol{B}_{\mathrm{I}}=B_{0}(z) \hat{\mathbf{z}}_{\mathrm{I}}+B_{1}(z)\left[\cos (\omega t) \hat{\mathbf{x}}_{\mathrm{I}}+\sin (\omega t) \hat{\mathbf{y}}_{\mathrm{I}}\right]
$$

where $B_{1}(z) \equiv 0$ for $z<z_{1}$ and $z>z_{2}$, and with $B_{0}(z)$ monotonic on $\left[z_{1}, z_{2}\right]$. In a frame rotating about the $z$-axis with angular frequency $\omega$ (frame II), the total magnetic field appears as (taking the $\hat{\mathbf{x}}_{I I}$-axis along $\left.B_{1}\right)^{10}$

$$
\boldsymbol{B}_{\mathrm{II}}=\left(B_{0}(z)+\frac{\omega}{\gamma}\right) \hat{\mathbf{z}}_{\mathrm{I}}+B_{1}(z) \hat{\mathbf{x}}_{\mathrm{II}}
$$

so that in frame II the angle between $B_{\mathrm{II}}$ and the $z$-axis is given by (see Fig. 2)

$$
\tan \theta=\frac{B_{1}(z)}{B_{0}(z)+\frac{\omega}{\gamma}} .
$$

Considering a location $z_{r} \in\left(z_{1}, z_{2}\right)$, where $\omega=-\gamma B_{0}\left(z_{r}\right)$ (i.e., the rotation frequency of $B_{1}$ matches a spin's Larmor precession frequency in $B_{0}$ ), and noting that $z_{r}$ must be unique, we see that so long as $B_{1}(z) \neq 0$ until such time as $\left(B_{0}(z)+\frac{\omega}{\gamma}\right)$ $\gg B_{1}(z)$, the field $\boldsymbol{B}_{\mathrm{II}}$ will rotate through $180^{\circ}$ for complete passage in either direction through the non-zero $B_{1}$ region. This occurs because while Eq. (4) demands that $B_{\text {II }} \| \hat{\mathbf{z}}_{\mathrm{I}}$ far enough from $z_{r}$ on either side of the resonance (since $B_{1}=0$ for $z<z_{1}$ and $z>z_{2}$ ), the denominator must change sign as the spin passes through the point $z_{r}$ where $B_{0}=-\omega / \gamma$. If this rotation of $\boldsymbol{B}_{\text {II }}$ occurs slowly enough, the neutron spin will adiabatically follow $\boldsymbol{B}_{\mathrm{II}}$, resulting in a $\pi$-flip since $\boldsymbol{B}_{\mathrm{II}}$ begins and ends parallel to the $\hat{\mathbf{z}}_{\mathbf{I}}$-axis but must rotate through $180^{\circ}$. The direction of travel simply changes whether $\boldsymbol{B}_{\mathrm{II}}$ is initially parallel or anti-parallel to the $z$-axis (and the spin), but not the fact that the spin is rotated through $180^{\circ}$. Fig. 3 shows the behavior of $\theta(z)$ for a single pass through an adiabatic fast passage device with a spatially varying $B_{1}$-field profile.

The parameters which affect whether the change in $\boldsymbol{B}_{\mathrm{II}}$ is slow enough to maintain adiabaticity are the speed of passage and the gradient of $B_{0}$, since both control the rate at which $\boldsymbol{B}_{\mathrm{II}}$ rotates, and the magnitude of $B_{1}$, since intuitively larger fields might be imagined to hold spins more effectively. In order to see how these parameters enter the problem explicitly we may follow Robiscoe ${ }^{12}$ (although here we have taken frame I as the lab frame, which simply amounts to an additional phase) and solve the Schrödinger equation for the wavefunction of a spin- $\frac{1}{2}$ particle. This is most easily done by transforming to a system (frame III) in which the $\hat{\mathbf{z}}_{\mathrm{III}}$-axis is along $\boldsymbol{B}_{\mathrm{II}}$. In this frame, where the spin simply precesses about $\boldsymbol{B}_{\mathrm{II}}$ with $\boldsymbol{B}_{\mathrm{II}}$ fixed in direction, the dynamics of the spinor components are 


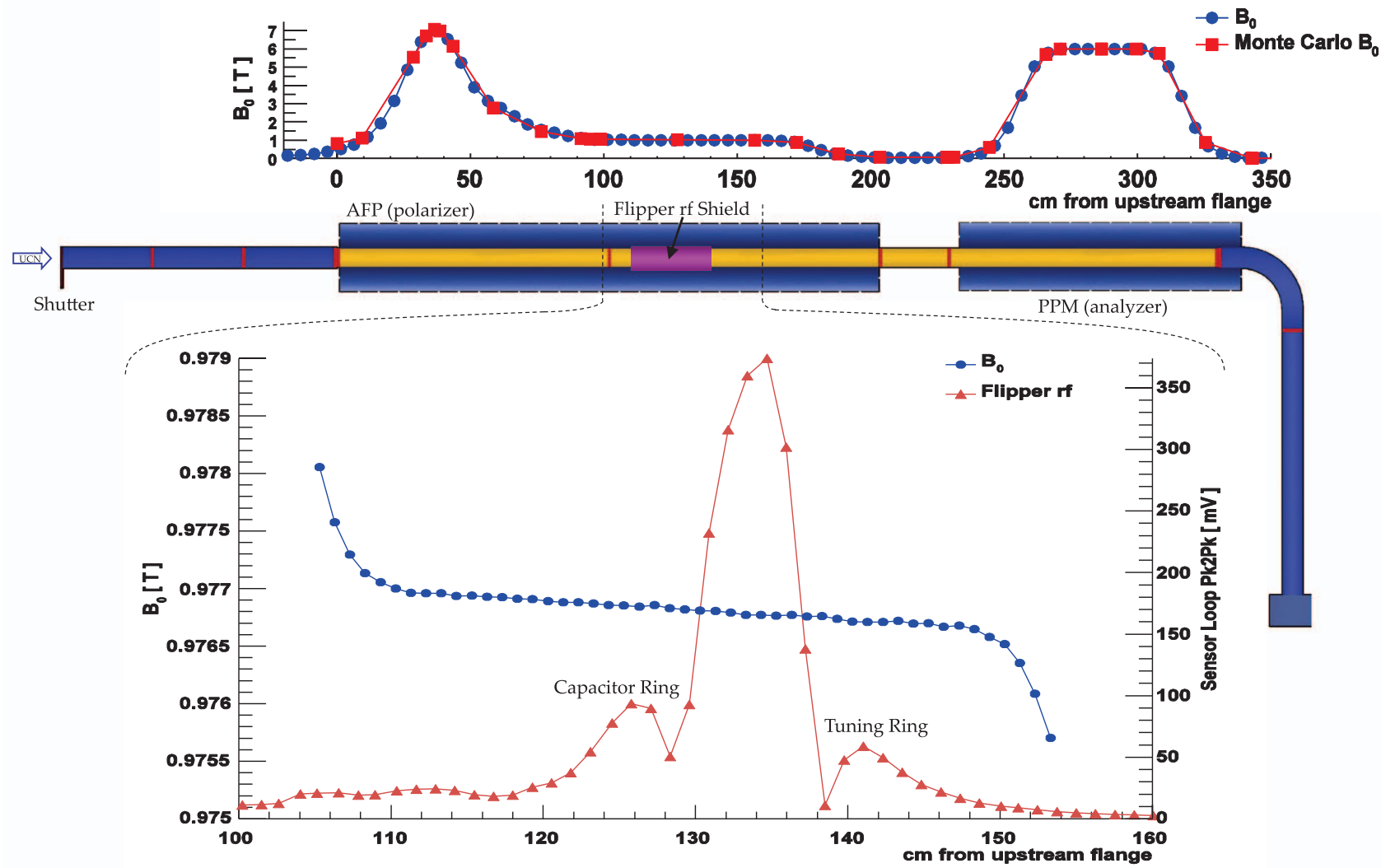

FIG. 1. (Upper) A scale drawing of the experimental geometry showing the longitudinal $B_{0}$-field magnitude (calculated by scaling and combining profiles measured individually for the main coil and each shim coil) with the linearized approximation used for Monte Carlo calculations superimposed. Dark shading indicates electropolished stainless steel guides and light shading indicates DLC-coated quartz guides. (Lower) An enlarged view of the precision gradient $1 \mathrm{~T}$ region for $0.1 \mathrm{G} / \mathrm{cm}$ shim coil settings with the longitudinal rf field profile superimposed.

given by

$$
\left(\begin{array}{c}
\dot{s}_{\mathrm{III}}^{+} \\
\dot{s}_{\mathrm{III}}^{-}
\end{array}\right)=\frac{i \gamma}{2} B_{\mathrm{II}}\left(\begin{array}{c}
s_{\mathrm{III}}^{+} \\
-s_{\mathrm{III}}^{-}
\end{array}\right)+\frac{\dot{\theta}}{2}\left(\begin{array}{c}
s_{\mathrm{III}}^{-} \\
-s_{\mathrm{III}}^{+}
\end{array}\right),
$$

where

$$
B_{\mathrm{II}}(t)=\sqrt{\left(B_{0}(t)+\frac{\omega}{\gamma}\right)^{2}+B_{1}^{2}(t)}
$$

and

$$
\dot{\theta}=\gamma \frac{-\gamma B_{1} \dot{B}_{0}+\dot{B}_{1}\left(\omega+\gamma B_{0}\right)}{\left(\omega+\gamma B_{0}\right)^{2}+\left(\gamma B_{1}\right)^{2}},
$$

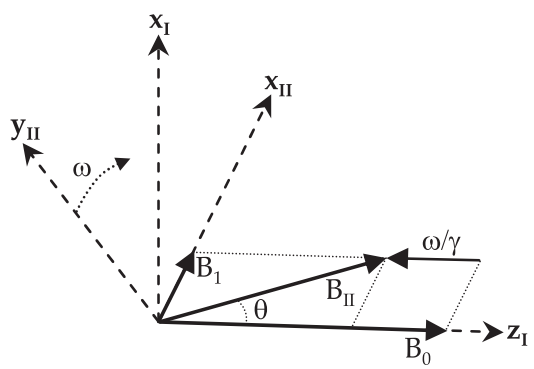

FIG. 2. $\boldsymbol{B}_{\mathrm{II}}$ is the total field observed in a frame rotating with angular frequency $\omega$ (frame II), which results from the superposition of a static field $\left(\boldsymbol{B}_{0}\right)$ and a rotating field $\left(\boldsymbol{B}_{1}\right)$ in the laboratory frame (frame I). with the time dependence in $B_{0}(t)$ and $B_{1}(t)$ due to the spatial dependence of the field coupled to the (classical ballistic) motion of the neutron. The first term in Eq. (5), which does not mix the spinor components, simply evolves the spin's precession around $B_{\mathrm{II}}$; if it dominates the evolution then the spin state will remain fixed relative to the $\hat{\mathbf{z}}_{\mathrm{III}}$-axis (i.e., $\boldsymbol{B}_{\mathrm{II}}$ ), and hence the spin will be flipped upon passage through the $B_{1}$ field region since the direction of the $\hat{\mathbf{z}}_{\text {III }}$-axis rotates through $\pi$. The second term, on the other hand, mixes the spinor components, and so can lead to non-preservation of the spin state, i.e., to the spin not being flipped. It is reasonable, then, to define the adiabaticity parameter

$$
\alpha=\frac{|\gamma| B_{\mathrm{II}}}{|\dot{\theta}|}
$$

as the ratio of the magnitude of the two terms in Eq. (5). When the first term dominates, i.e., when the Larmor precession frequency about the field observed from frame II is very large compared to the rate at which that field is changing $(\alpha \gg 1)$, a spin flip is very likely. On the other hand, if the Larmor precession frequency about $B_{\mathrm{II}}$ is small compared to the rate of change of $B_{\mathrm{II}}$ (the second term dominates), a spin flip is unlikely.

A simple analytic expression for the spin flip efficiency $\epsilon$ may be obtained under the assumption that passage through the $B_{1}$-field region happens with $\alpha=$ const. Re-writing 

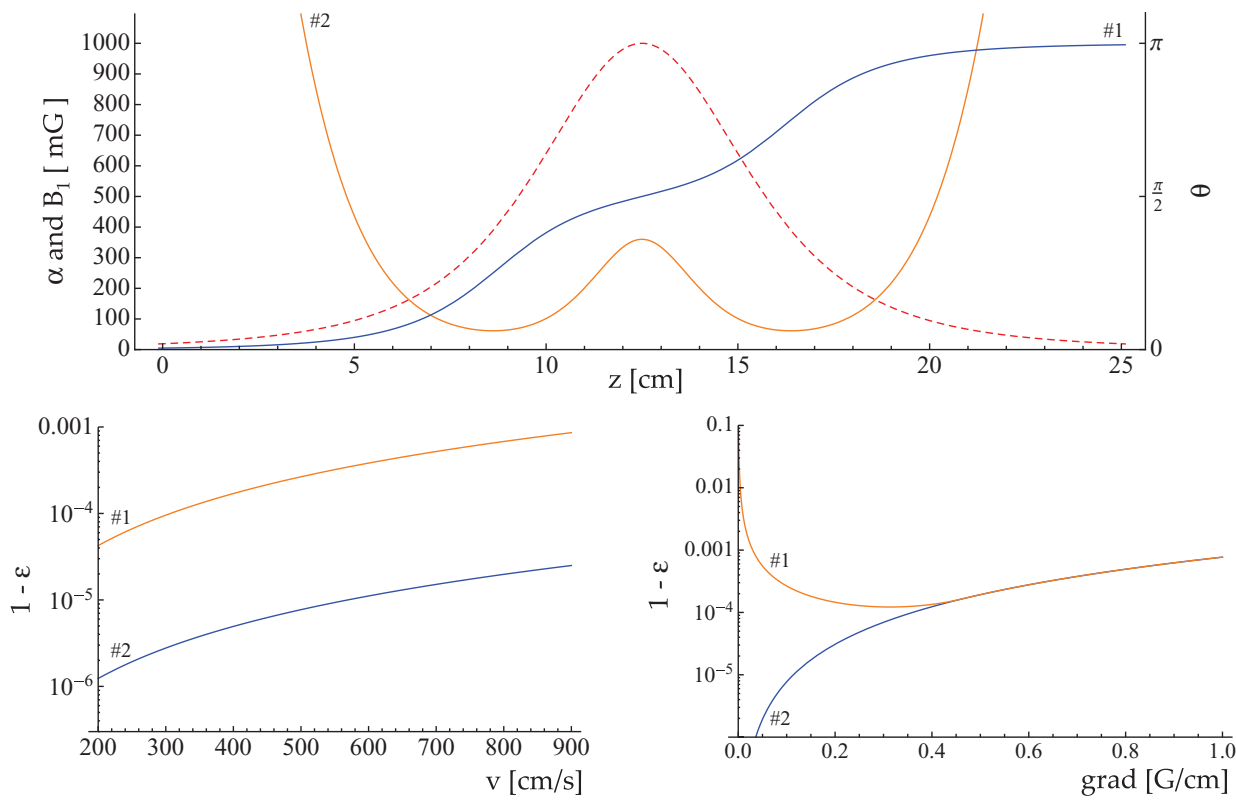

FIG. 3. (Upper) Angle relative to the z-axis made by the total field as observed from the rotating system (blue, \#1) for a $B_{0}$ gradient of $0.1 \mathrm{G} / \mathrm{cm}$ and a varying $B_{1}$-field profile (dashed plot) centered on the point where $B_{0}=-\omega / \gamma$. Superimposed is a plot of the adiabaticity parameter $\alpha$ under the same conditions for a neutron with a longitudinal speed of $500 \mathrm{~cm} / \mathrm{s}$ (orange, \#2). (Lower left) Inefficiency envelopes predicted by assuming a constant $\alpha$ equal to $\alpha_{\min }$ for a constant $B_{1}=1 \mathrm{G}$ case (blue, \#2) and for the varying $B_{1}$ case depicted in the upper plot (orange, \#1). (Lower right) Spin flip inefficiency calculated in the constant- $\alpha$ case as a function of $B_{0}$ gradient for $500 \mathrm{~cm} / \mathrm{s} \mathrm{UCN}$ experiencing the constant $B_{1}$ field (blue, \#2) and the varying $B_{1}$ field (orange, \#1).

Eq. (5) in terms of the independent variable $\theta$ leads to ${ }^{12}$

$$
\epsilon=1-\frac{1}{\kappa^{2}} \sin ^{2}\left(\frac{\pi}{2} \kappa\right)
$$

with

$$
\kappa=\sqrt{1+\alpha^{2}}
$$

so that in this special case the spin flip efficiency $\epsilon$ is always greater than or equal to $1-1 / \kappa^{2}$. Considering for a moment the case of a constant $B_{0}$ gradient and a constant value of $B_{1}$ (assumptions incompatible with a constant $\alpha$ ), it follows from Eq. (6) that $B_{\mathrm{II}}$ takes on a minimum value at resonance, i.e., at the point $z_{r}$. On the other hand, Eq. (7) dictates that $\dot{\theta}$ is maximum at resonance. As a result, the adiabaticity parameter $\alpha$ must necessarily be a minimum there. Selecting this on-resonance value of $\alpha$ as the value to be used in Eqs. (9) and (10), and assuming the $B_{0}$ gradient is small enough that changes in the speed $v$ with which the spin traverses the $B_{1}$-field region may be neglected (an important consideration for UCN) so that $\dot{B}_{0}=\left(\nabla_{z} B_{0}\right) v$, we obtain

$$
\epsilon>1-\frac{\left(\nabla_{z} B_{0}\right)^{2} v^{2}}{\left(\nabla_{z} B_{0}\right)^{2} v^{2}+\gamma^{2} B_{1}^{4}} .
$$

The efficiencies predicted by Eq. (11) (or more generally by Eq. (9) in the non-constant $B_{1}$ case where the minimum $\alpha$ does not necessarily occur at the resonance point) are expected to provide a lower bound for the actual spin flip efficiency and may be used as a figure of merit for the evaluation of a particular adiabatic fast passage spin flipper system. The lower left panel of Fig. 3 provides some examples of inefficiency envelopes calculated in this way. Note, however, that when the $B_{1}$ field has a strong spatial variation, care must be utilized when applying Eq. (11) as a figure of merit. In particular, while Eq. (11) makes it appear that high spin flip efficiencies are correlated with small $B_{0}$ gradients, in the case of a spatially varying $B_{1}$ field, where the minimum $\alpha$ may be separated from the resonance point, Eq. (7) indicates that decreasing the gradient too much can cause a decrease in the flipping efficiency. The lower right panel of Fig. 3 demonstrates this effect. Nevertheless, for a suitable $B_{0}$-field gradient and sufficiently large rotating fields, Eq. (11) is expected to capture the scaling of the spin flip efficiency and suggests that the flipping efficiency for an adiabatic fast passage scheme may be made quite high over a wide band of neutron speeds. In fact, practical adiabatic fast passage spin flippers have been constructed for use with UCN as well as thermal and cold neutron beams. ${ }^{13}$ Reported efficiencies for such cold neutron flippers are $\bar{\epsilon}=0.99 \pm 0.01$ for a device used at the Nuclear Physics Institute in Saint-Petersburg (PNPI) in, among other things, neutron decay angular correlation measurements with cold neutrons, ${ }^{13}$ and $\bar{\epsilon}=0.9993$ (1) (stat) for the flipper used with the PERKEO II spectrometer, ${ }^{14}$ in which the highest precision measurement of the $\beta$ asymmetry to date using cold neutrons was performed.

\section{Theory of the birdcage coil}

UCN are particularly amenable to adiabatic spin flipping due to their low characteristic speed (cf. Eq. (11)), so that the success of the PNPI and PERKEO II cold neutron spin flippers suggests that an adiabatic fast passage spin flipper would be ideal for the UCNA experiment. The PNPI and PERKEO 
II flippers, however, operate in $B_{0}$ fields of a few $\mathrm{mT}$, requiring operational frequencies in the $\mathrm{kHz}$ range. In the UCNA experiment, on the other hand, the fact that field gradients can substantially alter the UCN kinetic energy in a spin dependent way must be considered when selecting the $B_{0}$ field for the flipping region, which sits between the typical $1 \mathrm{~T}$ longitudinal field needed to transport electrons out of the decay volume and a longitudinal $7 \mathrm{~T}$ polarizing field. ${ }^{8}$ For that reason, the solenoidal polarizing magnet (called the AFP mag$n e t$ ) was designed to provide a spin flipping region with an ambient field of $1 \mathrm{~T}$ so that the UCN kinetic energy in the decay volume would be the same for both spin states. One consequence of this choice is that the UCNA spin flipper must operate near $29 \mathrm{MHz}$, a frequency large enough that efficient devices become necessary in order to produce reasonable $B_{1}$ field strengths with minimal rf input power. The UCNA spin flipper is therefore based on a rf cavity, called a birdcage coil resonator, proposed initially by Hayes in 1985 for use in medical magnetic resonance imaging (MRI), ${ }^{15}$ and which has since become ubiquitous in both MRI and NMR technology due to its high efficiency (implying an excellent signal-to-noise ratio in receive mode) and superior field homogeneity.

The design of a birdcage resonator is predicated on the observation that a sinusoidal distribution of surface current

$$
\boldsymbol{\sigma}(\phi, z)=\sigma_{0} \sin (\phi) \hat{\mathbf{z}}
$$

on a cylindrical surface $S$ produces a transverse field everywhere inside $S$ given by

$$
\boldsymbol{B}(r, \phi, z)=B_{\mathrm{t}} \hat{\mathbf{x}}
$$

where the $+x$-axis is in the radial direction and passes through $\phi=0$. Varying this current density sinusoidally in time allows the field to be decomposed as

$$
\begin{aligned}
\boldsymbol{B}=B_{\mathrm{t}} \sin (\omega t) \hat{\mathbf{x}}= & \frac{B_{\mathrm{t}}}{2}[\{\sin (\omega t) \hat{\mathbf{x}}+\cos (\omega t) \hat{\mathbf{y}}\} \\
& -\{\sin (-\omega t) \hat{\mathbf{x}}+\cos (-\omega t) \hat{\mathbf{y}}\}],
\end{aligned}
$$

i.e., as two fields of magnitude $B_{\mathrm{t}} / 2=B_{1}$ rotating in opposite directions with angular frequency $\omega$. Surrounding a longitudinal static field with this sinusoidally varying current density therefore provides the requisite fields for adiabatic spin flipping as discussed in Sec. I B, albeit with an additional $B_{1}$ field rotating with angular frequency $-\omega$. As Eq. (3) shows, however, as long as $B_{1}$ is small compared to $B_{0}$, this counterrotating field will be so far off resonance as to produce virtually no effect. (Specifically, the counter-rotating field produces a relative shift in the resonance frequency of $\left(\frac{B_{1}}{2 B_{0}}\right)^{2}$, the Bloch-Siegert shift, as well as the addition of higher order resonances for $\omega=\frac{\gamma B_{0}}{2 n+1}, n$ an integer. ${ }^{10}$ )

A birdcage coil creates a discretized approximation to the surface current of Eq. (12) by evenly spacing $N(N$ even) straight parallel conductors called rungs around the surface of a cylinder (see Fig. 4) and providing coupling between neighboring rungs to produce $N$ meshes. There are a number of approaches to coupling the rungs together, but although other possibilities exist they generally fall into two categories: low-pass and high-pass. Low-pass designs feature

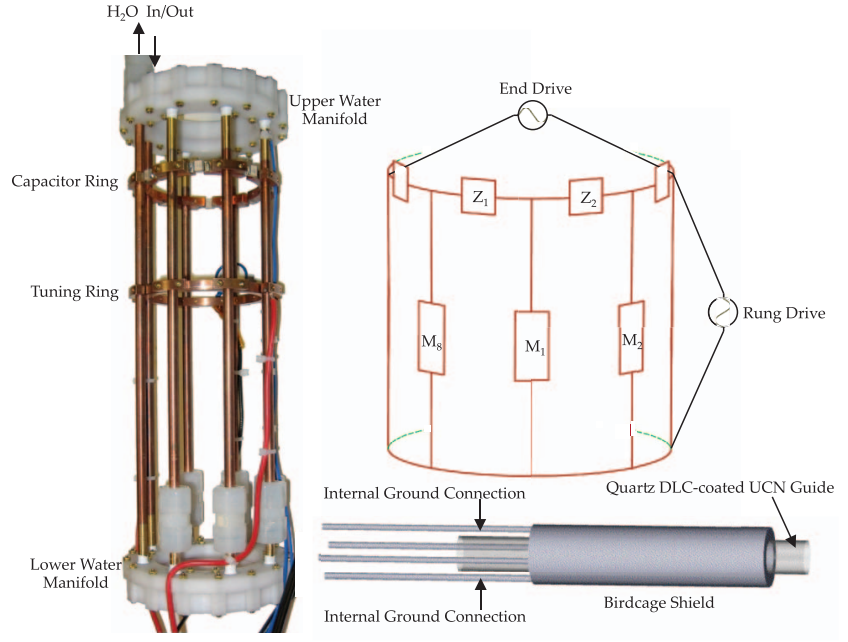

FIG. 4. The eight-rung high-pass UCNA birdcage resonator (left), a lumpedelement circuit model indicating connections for end- and rung-driving schemes (right), and the birdcage rf shield (bottom) are shown. The impedance $Z_{i}=\frac{1}{i \omega C_{i}}+i \omega L_{i}$ (representing a capacitor and inductor in series), where $C_{i}$ corresponds to the capacitance of the capacitor connected physically between the rungs, $L_{i}$ represents the self-inductance of the $i$ th capacitor ring element, and $\omega$ is the driving frequency. $M_{i}$ represents a combination of the rung self-inductance and mutual inductance between adjacent meshes.

conducting end-rings at the top and bottom of the coil to which the rungs are capacitively coupled. High-pass designs, on the other hand, capacitively couple each rung at the top (or at both the top and bottom) to its two nearest-neighbor rungs (forming a capacitor ring). In either case the connected structure is resonant, sustaining a sinusoidal standing wave when driven at the appropriate frequency.

A lumped-element model is helpful for understanding how such a device produces the required current distribution. Fig. 4 presents such a model for an eight-rung highpass birdcage coil similar to the UCNA resonator, where the impedances $Z_{i}$ are taken to be a series combination of a capacitance $C_{i}$ (corresponding to the capacitor connected physically between rungs) and an inductance $L_{i}$ (corresponding to the self-inductance of a capacitor ring element). The rung inductances $M_{i}$ account for both the rung's self-inductance and the mutual inductance between the $(i-1)$ st and $i$ th meshes. Perfect fidelity is not possible with the simple diagram in Fig. 4, and work has been done on calculating the effect of more detailed couplings in such a geometry. ${ }^{16}$ For the present purposes, however, the model of Fig. 4 suffices despite the fact that it explicitly ignores, for example, flux coupling between non-adjacent meshes as well as any flux coupling between end-ring elements. Thinking of the circuit in Fig. 4 for the moment as a transmission line formed by a series of identical $\pi$-sections, and noting that each $\pi$-section will impart a phase shift $\varphi_{0}(\omega)$ to a traveling wave of angular frequency $\omega,{ }^{17}$ if $N \varphi_{0}$ equals a multiple of $2 \pi$, the phase of a wave at the input of the line will be equal to the phase at the exit; since the birdcage geometry has the input and output physically connected, a standing wave with one wavelength spanning the device will be created for an appropriate driving frequency. Such a standing wave will produce a current distribution in 
the rungs which at each instant of time approximates the continuous distribution of Eq. (12).

Since the design of a birdcage resonator will generally have some specific frequency target for the fundamental (or NMR-useful) modes (29.2 MHz in the case of UCNA), and since exciting modes other than the fundamental is to be avoided, it is useful to know the resonance spectrum in terms of the model parameters. A mesh analysis is fruitful in this regard since it lends itself to understanding the effects of broken symmetry in an actual device. Tropp ${ }^{18}$ carries out such an analysis for a low-pass birdcage. The high-pass case is similar, resulting in a spectrum

$$
\omega_{j}=\frac{1}{\sqrt{C\left[2 M+L-2 M \cos \frac{2 \pi j}{N}\right]}} \quad(j=1, \ldots, N),
$$

where we have assumed a symmetric network, i.e., $C_{i}=C$, $M_{i}=M$, and $L_{i}=L$ for all $i$. Of these $N$ normal modes there are $N / 2-1$ degenerate pairs

$$
\{1, N-1\}, \ldots,\{N / 2-1, N / 2+1\}
$$

corresponding to the two possible directions of wave propagation, plus two non-degenerate modes $\{N / 2, N\}$. It is the fundamental modes $j=\{1, N-1\}$ which create the discretized current variation we desire, and these NMR-useful modes possess the highest frequency of all the degenerate modes. (In a low-pass resonator the NMR-useful modes appear at the smallest degenerate-mode frequency.)

Since the actual realization of a birdcage resonator will not always be perfectly symmetric, i.e., each mesh will not necessarily be electrically equivalent, it is helpful during the design process to have some idea of what to expect in the nonsymmetric case. As the physical capacitors might be expected to provide the largest source of an asymmetry, especially in directly driven coils, it is reasonable to consider a perturbation of the $k$ th capacitor

$$
C_{k} \rightarrow C_{k}(1-\delta)
$$

The first-order shifts in eigenfrequency are found to be

$$
\begin{aligned}
& \left\{\begin{array}{l}
\Delta \omega_{j}=0 \text { for } j=1,2,3, \ldots, \frac{N}{2}-1 \\
\Delta \omega_{j}=\omega_{j}\left(\frac{1}{\sqrt{1-\delta / 4}}-1\right)
\end{array}\right. \\
& \quad \text { for } j=\frac{N}{2}+1, \frac{N}{2}+2, \ldots, N-1 .
\end{aligned}
$$

Importantly, the perturbation does not disrupt the current distribution to first order (see Tropp ${ }^{18}$ for a discussion of the effect on the current distribution in second order), but it does fix the polarization of the modes, i.e., the physical location of the current nodes. So, a non-symmetric $N$-rung birdcage coil is characterized by $N$ non-degenerate modes with the two NMR-useful modes $\{1, N-1\}$ being close in frequency and having their current nodes separated by $90^{\circ}$ so that they produce transverse magnetic fields at right angles to each other.

\section{THE UCNA SPIN FLIPPER}

\section{A. Physical construction of the birdcage resonator}

The physical design of the UCNA spin flipper was predicated on several operational requirements, the primary one being the capability of producing transverse fields approaching $5 \mathrm{G}$ (i.e., a $B_{1}$ field of $2.5 \mathrm{G}$ ), which Eq. (11) indicates leaves a good safety margin for $99.9 \%$ efficient spin flipping of $8 \mathrm{~m} / \mathrm{s}$ neutrons in a $0.6 \mathrm{G} / \mathrm{cm} B_{0}$-field gradient (the nominal gradient in the $1 \mathrm{~T}$ region of the AFP magnet without utilizing shim coils). This specification, in conjunction with a required operational frequency near $29 \mathrm{MHz}$, meant that the device would likely need to tolerate several hundred watts of input power. As a result, a high-pass design utilizing 1/4 in. $\mathrm{Cu}$ tubing for the rungs and solid $\mathrm{Cu}$ segments for the capacitor ring connections was selected to allow for easy water cooling and to provide adequate thermal mass. The rungs were held in place by nylon manifolds which allowed chilled water to be introduced into one rung, flow serially through the intervening rungs, and then be extracted at the final rung (see Fig. 4). Since maximizing the rf field seen by the neutrons for a particular input power necessitates holding the $\mathrm{Cu}$ tubes close to the neutron guide, the plastic water-cooling manifolds were designed to fit closely around a $6.985 \mathrm{~cm}$ OD diamond-like carbon (DLC)-coated quartz neutron guide tube. This resulted in the $\mathrm{Cu}$ tubes forming a cavity of diameter $8.74 \mathrm{~cm}$.

As Eq. (15) shows, the frequency of the fundamental mode in a birdcage is set by the value selected for the capacitors as well as by the rung and capacitor ring inductances. Given a required operating frequency, a value for the capacitors is typically chosen to get as close as possible to the desired resonant frequency, and then fine tuning of the fundamental is accomplished by changing the distance between the end-rings, thereby changing the value of $M$. Often high-pass birdcage coil designs used for NMR feature capacitor rings on both ends of the birdcage, which enhances longitudinal field homogeneity. However, since capacitor rings in prototype models of the UCNA spin flipper turned out to be difficult to move along the rungs without damaging the solder joints, the UCNA birdcage has an in-practice fixed capacitor ring and a movable, solid $\mathrm{Cu}$ tuning ring clamped to the rungs. $820 \mathrm{pF}$ capacitors were chosen for the capacitor ring to yield a cavity (after adjustment of the tuning ring position) whose length was approximately twice its diameter. Keeping the volume of the cavity small had the advantage of enhancing the field produced for a given input power as well as limiting the distance over which the $B_{0}$-field gradient needed to be controlled. The downside of a "short" cavity was in reduced homogeneity of the $B_{1}$ field, but as rf field homogeneity was of secondary importance compared to field strength the tradeoff was deemed acceptable.

The number of rungs used in the construction of a birdcage is an important design criterion as well. In particular, the number of rungs affects rf field homogeneity since having more rungs enhances the fidelity of the discretized approximation to the current distribution of Eq. (12) provided by the coil. Increasing the number of rungs, however, also increases the number of lumped circuit elements and therefore energy loss in the device. There seems 
to be no consensus in the literature as to an ideal number of rungs since birdcage coils used in NMR are built for a wide variety of frequencies, on a wide variety of scales, and for a number of different drive arrangements. Crozier et al. ${ }^{19}$ studied the effect that varying the number of rungs in high-pass birdcage resonators with dimensions similar to the UCNA birdcage had on the coil quality factor $\mathrm{Q}$, defined as $2 \pi \times$ (energy stored / (energy lost per cycle). It was found that birdcages with 12 and 16 rungs had Q-values roughly half of those measured in 8-rung designs. Although the birdcages in the Crozier study were tuned to resonate at ten times the frequency of interest for UCNA (as well as driven differently), prototype UCNA birdcages of 8 and 16 rungs demonstrated a similar trend, with the 16-rung versions producing about half the rf field of the 8 rung varieties. As a result, rf field magnitude was again selected over homogeneity for the UCNA birdcage, which was built with eight rungs.

In order to further minimize energy loss, high-Q porcelain multilayer capacitors from American Technical Ceramics (ATC100C821GMN1000XC), which are designed for high power rf applications, were used in construction of the UCNA spin flipper. It was possible to obtain these capacitors with microstrip leads, which facilitated their connection between the solid $\mathrm{Cu}$ elements forming the bulk of the capacitor ring. Actual assembly of the birdcage was relatively straightforward, although care was required to produce robust and consistent solder joints between the capacitors and the $\mathrm{Cu}$ capacitor ring elements. Since the capacitor rings were somewhat fragile when unsupported, the rungs were first connected to the water cooling manifolds, the tuning ring installed to correctly align the rungs, and then the capacitor ring was assembled in place on the rungs. Soldering was performed with a resistance-soldering station and with the upper manifold immersed in water to prevent heat damage to the plastic. Following assembly of the capacitor ring and with the tuning ring removed, the capacitance across each section of the birdcage was measured with a standard DVM in order to check for any electrical asymmetries. Any section showing a variation from the nominal value of $0.96 \mathrm{nF}$ had its capacitor replaced. This process was repeated until each section's capacitance agreed to within the precision of the DVM $(\sim \pm 0.005 \mathrm{nF})$.

\section{B. Balanced drive and the rf shield}

It is generally observed that a closely coupled rf shield around a birdcage dramatically affects the performance of the coil by, for example, reducing the Q-value of the system and causing shifts in the resonance spectrum. ${ }^{20,21}$ Since the birdcage unit (outer diameter of $8.74 \mathrm{~cm}$ ) must fit inside the AFP magnet bore (inner diameter $12.7 \mathrm{~cm}$ ), the presence of the bore would certainly alter the operation of the coil, necessitating in situ tuning. The addition of a grounded rf shield remedied this problem, not only allowing for stable, consistent operation regardless of the surrounding environment, but also preventing contamination of beta detector signals with rf noise.

Fig. 4 shows the cylindrical Al $\mathrm{rf}$ shield used for the UCNA spin flipper, which has an outer diameter of $12.065 \mathrm{~cm}$ (wall thickness $0.159 \mathrm{~cm}$ ). The four legs connect via bellows to the exterior of the AFP magnet bore, providing conduits for the power lines, connections to a $\sim 2 \mathrm{~cm}$ diameter internal sensor loop which sits flush against the outside of the cavity, connections to a resistance temperature detector (Omega Engineering model\# RTD-2-F3102-180-T-B), and water cooling lines. Since the AFP magnet bore is kept under vacuum, the rf shield creates a vacuum seal against the UCN guide passing through the spin flipper. This allows the inside of the shield and thus the birdcage coil to remain at atmosphere, which significantly aids in cooling the device and mitigates the possibility of arcing. Bench tests of this shield configuration at $100 \mathrm{~W}$ of input power found the maximum rf power radiated from the device at $2.5 \mathrm{~cm}$ to be $1 \mathrm{~mW} / \mathrm{cm}^{2}$, while the radiated rf power detected at the exit of the AFP magnet bore with the spin flipper in place was an order of magnitude less.

The quality of the rf shield's ground was important to ensure stability and limit the leakage of rf radiation. In particular, it was found that driving the shielded birdcage in a balanced mode (i.e., where the two drive lines are forced to be $180^{\circ}$ out of phase) was imperative since unbalanced drive resulted in a system that was quite sensitive to the environment. In the case of the UCNA spin flipper, balanced drive was accomplished via a Werlatone $180^{\circ}$ hybrid junction (model H1484-10) with the difference port (J2) of the junction fed by an amplified rf signal and the sum port (J1) connected to a $50 \Omega$ terminator (see Fig. 6). RG-142 coaxial cable carried the resulting signal from the $\mathrm{J} 3$ and $\mathrm{J} 4$ ports of the junction, with the center conductors of the two drive lines connected directly to the birdcage while the two braids formed the rf ground and were connected to the rf shield using $\mathrm{CuBe}$ fingerstock at two points inside opposite legs of the rf shield $43 \mathrm{~cm}$ from the ends of the cables.

\section{Power coupling}

The question of how best to deliver power to a birdcage coil is important since forcing the system sets boundary conditions that determine which portions of the spectrum described previously are actually excited. A variety of approaches are used in practice, which include both direct application of power across some part of the coil and inductive coupling schemes. Given the space and shielding constraints imposed on the UCNA spin flipper by the experimental geometry, a direct power coupling approach was judged to be the simplest, but a clear choice of how best to couple power directly to the coil was not readily evident in the literature. Two typical approaches include rung-drive, where power is applied across a rung, and end-drive, where power is applied between two points on the capacitor ring. Rung-drive appears advantageous because a rung is driven directly, ensuring maximal rung currents (and thus field magnitude). End-drive, on the other hand, especially in the case of a balanced drive connected to the appropriate rungs $180^{\circ}$ apart, naturally selects a single fundamental mode (in a non-symmetric birdcage), thereby reducing the coupling of energy into other modes.

In order to compare these two particular drive schemes, a nodal analysis of the network is convenient since in both 

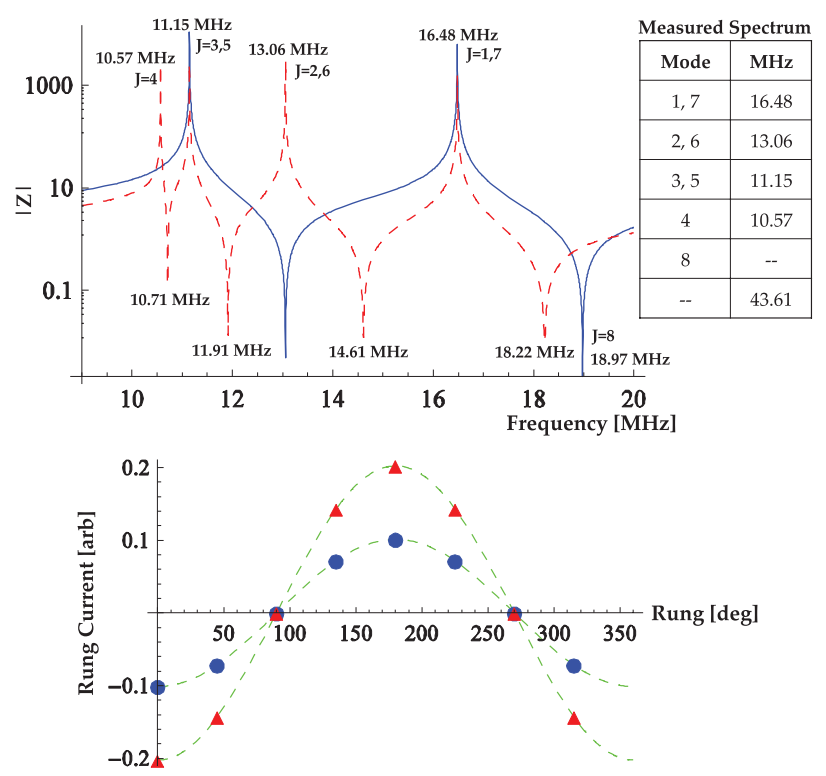

FIG. 5. (Top) Comparison of theoretical end-drive (blue) and rung-drive (red/dashed) birdcage spectra for the circuit in Fig. 4 with $\mathrm{C}=820 \mathrm{pF}, \mathrm{L}$ $=85.8 \mathrm{nH}$, and $\mathrm{M}=47.7 \mathrm{nH}$, where $\mathrm{L}$ and $\mathrm{M}$ were determined by fitting the measured spectrum (inset table), determined via inductive coupling, with Eq. (15). (It is presently not known if the resonance seen at $43.61 \mathrm{MHz}$ possessed a current distribution appropriate for the $j=8$ mode.) (Bottom) A comparison of the theoretical rung current distributions for rung-drive (red triangles) and end-drive (blue circles) at the NMR-useful mode frequency $16.48 \mathrm{MHz}$.

cases the birdcage is driven by the direct application of voltage to specific points in the circuit. The spectra resulting from such an analysis applied to the circuit in Fig. 4 with the two indicated drive connections are compared with each other and with the undriven spectrum in Fig. 5. As can be seen, enddrive allows for the excitation of all possible natural modes except $\mathrm{j}=4$, although the modes which occur at impedance minima do not appear to produce simple sinusoidal current distributions. Rung-drive, on the other hand, not only allows all natural modes except $\mathrm{j}=8$ to be excited, but several additional superposition modes (all impedance minima) can be excited as well. Fig. 5 also shows, as expected, that rung-drive produces larger rung currents (and thus a greater rf field) for the same input voltage.

According to Eq. (11), the characteristic of a birdcage coil used as an adiabatic fast passage neutron spin flipper which most directly affects the spin flip efficiency is the field strength. This would seem to argue for rung-drive since it produces the largest rung currents. In practice, however, bench testing of prototype spin flippers never demonstrated enhanced fields for rung-drive, and since overall the enddriven spectrum was cleaner and seemed generally more stable, the UCNA spin flipper was assembled using end-drive. In the final version of the birdcage, the center conductors of the two drive lines were connected via $\sim 25 \mathrm{~cm}$ of stranded 14 AWG wire to rungs $180^{\circ}$ apart. Connections were made by clamping the wires to the rungs using the tuning ring clamps since bench tests indicated an enhanced quality factor for connections made at the tuning ring rather than at the capacitor ring.

\section{Tuning the birdcage}

Since the fundamental modes of a non-symmetric birdcage have preferred polarizations, there should be a preferred orientation for exciting a fundamental mode (particularly for the chosen $180^{\circ}$ end-drive arrangement) in a real birdcage. The first step in tuning the UCNA birdcage, then, was to measure its spectrum, identifying the fundamental modes and ascertaining the optimum drive orientation needed to excite them. Since the presence of driving components necessarily distorts the spectrum, the native spectrum of the UCNA birdcage was measured using weak inductive coupling. This was done by exposing the center conductor at the end of a length of RG-142 coaxial cable and forming that conductor into a rectangle $(\sim 10 \mathrm{~cm} \times 4 \mathrm{~cm})$ with the end connected to the braid of the still-insulated section of cable. This formed an exciting antenna which was placed $2.89 \mathrm{~cm}$ from the birdcage (long sides parallel to the rungs) and connected to an HP4195A network analyzer supplemented by a power divider (Mini-Circuits ZFSC-2-1-N) and a directional coupler (MiniCircuits ZFDC-10-18-N) that together allowed measurement of the complex reflection coefficient $\Gamma$, which gives the ratio of power reflected from the input of the antenna to power incident on the antenna (magnitude squared) and the relative phase of the reflected and input signal, all as a function of frequency. Birdcage resonances were then identified with minima in the plot of $|\Gamma|$ versus frequency.

By rotating the birdcage about its symmetry axis in front of the antenna and observing the relative strengths of the excitations, an optimum driving orientation for one of the fundamental modes could be determined, characterized by the smallest value of $|\Gamma|$ for the mode of interest and, if the fundamental was split, a corresponding large value of $|\Gamma|$ for the split mode. The spectrum of the UCNA spin flipper (before final tuning ring adjustment) obtained in this manner is the one indicated in Fig. 5, which also provides values of the birdcage inductances obtained by fitting the spectrum with Eq. (15). Note that there is no resolvable splitting in the modes of the UCNA birdcage, indicating a high degree of symmetry; this was by no means always the case in prototype devices, and it is believed that improvements in consistency of assembly are responsible for this result. Birdcage orientations which put a rung directly in front of the antenna (the orientation which should place a current node on that nearby rung since oppositely directed currents are induced in the adjacent meshes) were found to excite the fundamental mode most strongly, indicating that drive points should be located at rungs rather than between them. Values of $|\Gamma|$ corresponding to the fundamental mode for these eight orientations differed by only 13\% (as opposed to previous cases where the modes were clearly split and some orientations did not excite one of the fundamental modes at all). Since for $180^{\circ}$ end-drive the current nodes are $90^{\circ}$ around the birdcage from the drive points, the ideal rungs to use for the drive connections are those $90^{\circ}$ away from the rung nearest the exciting antenna in the optimal orientation. However, since there was not a significant difference between the eight best orientations for the UCNA spin flipper, a qualitative bench test where changes in the spectrum were observed for different drive points was used to select 
the rungs for final power connections. Once the drive connection was made, the driven spectrum was measured with the network analyzer driving the hybrid junction and calibrated up to the $\mathrm{J} 3 / \mathrm{J} 4$ ports using two sets of calibration standards. The position of the tuning ring was then adjusted to produce a fundamental mode frequency of $\sim 29 \mathrm{MHz}$, which shifted to $28.6825 \mathrm{MHz}$ once the birdcage was placed in the rf shield. Driving was not observed to cause any splitting in the fundamental mode, indicating that either the drive connections did not significantly perturb the birdcage or that the balanced $180^{\circ}$ end-drive provided good isolation from a perpendicularly polarized mode.

\section{E. Impedance matching}

Providing impedance matching between the $50 \Omega$ environment of the drive system and the birdcage resonator is critical for ensuring maximum transfer of power to the coil and thus maximum rf field magnitude for a given input power. Impedance matching is also important for large input power since significant reflected power has the potential to damage rf amplifiers and a large voltage standing wave ratio (VSWR) can cause thermal damage to coaxial cables. Impedance matching is generally accomplished with variablereactance elements, which ideally should be used near the coil in order to minimize the length of transmission line subject to reflections, thereby minimizing power loss. ${ }^{22}$ This was not considered feasible for the UCNA spin flipper since the possibility existed that the matching elements would require adjusting in situ and the spin flipper itself is inaccessible once installed in the AFP magnet bore. As a result, the length of transmission line between the coil and matching elements $(\sim 3.38 \mathrm{~m})$ was chosen to be long enough that the elements could be positioned outside of the AFP magnet, and then adjusted so that the required matching elements would be capacitors (which typically have much higher quality factors than inductors) in the 5-500 $\mathrm{pF}$ range corresponding to the vacuum variable capacitors (Jennings Technology CSV1-500-0005) selected for the application. A balanced reversed L-section was used to perform the matching (see Fig. 6), with the capacitors mounted in a sealed $\mathrm{Al}$ enclosure connected to the rf ground. Some enhancement in the efficiency of the system is likely achievable by performing partial local matching as described by Rath ${ }^{22}$ (although connecting capacitors directly to the birdcage drive points could potentially split the fundamental, requiring the addition of trimmer capacitors to restore symmetry $^{23}$ ), but it is not currently implemented.

After connecting the matching circuit between the fully assembled shielded birdcage coil and the hybrid junction, the matching capacitors were tuned using the network analyzer calibrated up to the input of the matching circuit. For arbitrary settings of the matching capacitors, two frequencies producing a real reflectance coefficient were typically observed in the vicinity of the fundamental birdcage mode. The first corresponded to the minimum value of $|\Gamma|$, while the second corresponded to the frequency which produced a maximum field in the coil (measured with a pickup loop attached to a $50 \Omega$-terminated oscilloscope channel). The variable ca-

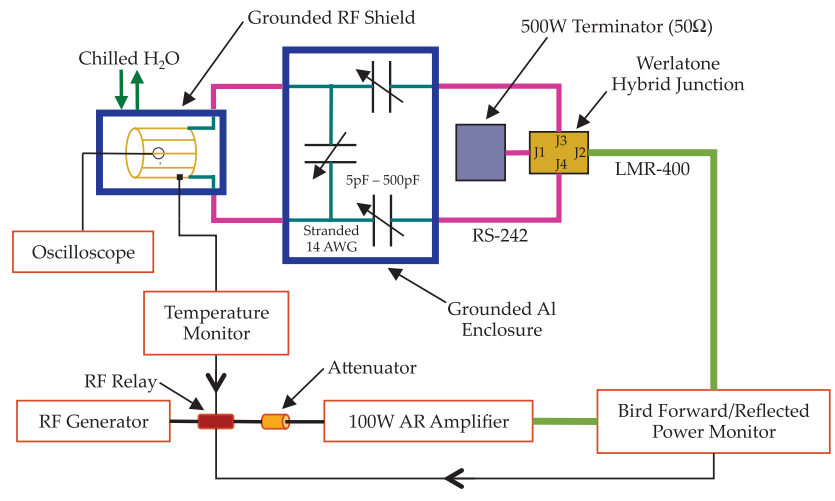

FIG. 6. Spin flipper system connections.

pacitors were adjusted so that the maximum-field frequency was matched to $50 \Omega$, leaving this as the only frequency near the fundamental which caused the reflectance coefficient to be real. Once the matching capacitors were set and the $\mathrm{Al}$ matching box sealed, the impedance match was quite robust against changes in the environment and relative positioning of the system elements, and the Q-value of the system (shielded coil plus matching elements) at the fundamental mode frequency of $28.525 \mathrm{MHz}$ was measured to be $\sim 300$.

\section{TESTING THE SPIN FLIPPER}

Testing of the UCNA spin flipper occurred just prior to the first measurement of the neutron $\beta$ asymmetry using UCN (Ref. 24) during the 2007 run cycle of Los Alamos National Laboratory's $800 \mathrm{MeV}$ proton accelerator, which was used to drive the solid deuterium $\left(\mathrm{SD}_{2}\right) \mathrm{UCN}$ source ${ }^{25}$ that provides UCN for the UCNA experiment. The goal of these studies was to evaluate the spin flipper's flipping efficiency by studying UCN transmission through a system comprised of a longitudinal polarizing field plus spin flipper (the crossed polarizer) followed by a second longitudinal field oriented identically to the first (the analyzer). In such a configuration, UCN which see the first polarizing field as an attractive potential and then have their spin reversed will see the second (analyzer) field as a retarding potential, resulting in an inverse correlation between the transmission through the system and the spin flip efficiency. Overall, there were three parts to the spin flipper tests: in situ characterization of the spin flipper system, measurement of the longitudinal UCN spectrum, and crossed polarizer analyzer measurements.

\section{A. Characterization of the spin flipper system}

The AFP magnet, which serves as the UCN polarizer and provides the required field environment for the spin flipper, was designed by American Magnetics (AMI) using a cryostat supplied by Ability Engineering mounted on a translation system which permitted $\sim 10 \mathrm{~cm}$ of motion both parallel and perpendicular to the magnet axis. The cryostat, shown in cutaway in Fig. 7, was constructed of 316 stainless steel, and the total mass of the cryostat, magnet, and stand was $929.86 \mathrm{~kg}$. The liquid $\mathrm{He}$ reservoir containing the superconducting 


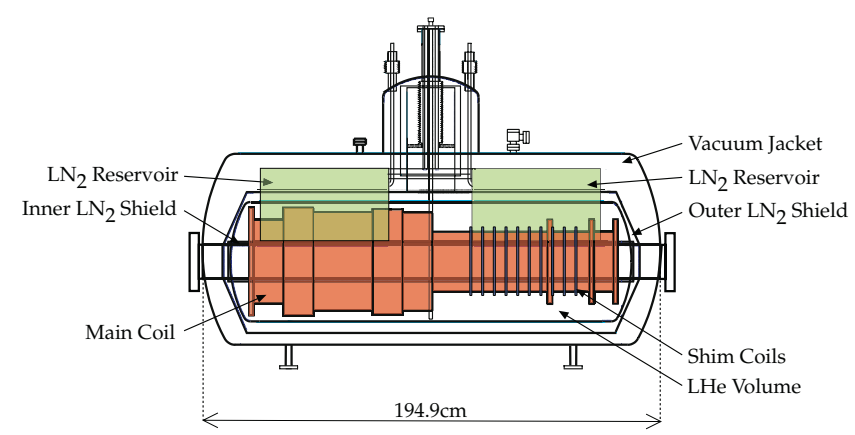

FIG. 7. Cutaway diagram of the AFP magnet cryostat. Maximum current in the main coil is $96.45 \mathrm{~A}$ which produces a maximum field of $7 \mathrm{~T}$ near the entrance (left side) of the magnet and a uniform $1 \mathrm{~T}$ field with a $\sim 0.6 \mathrm{G} / \mathrm{cm}$ gradient (right side). Shim currents from left (nearest the high-field region) to right calculated to produce the field profile shown in Fig. 1 were $0.864 \mathrm{~A}, 1.336 \mathrm{~A}, 4.000 \mathrm{~A}, 3.477 \mathrm{~A}, 3.561 \mathrm{~A},-1.104 \mathrm{~A},-3.231 \mathrm{~A}, 0.993 \mathrm{~A}$, $-0.978 \mathrm{~A}$, and $1.269 \mathrm{~A}$.

windings had a capacity of 2001 and was thermally shielded by superinsulation, inner and outer liquid nitrogen jackets coupled to a 461 reservoir, and an outer vacuum jacket. With the magnet energized, the He boiloff rate into the recovery and liquefaction system was about $4 \mathrm{l} / \mathrm{h}$. The $194.9 \mathrm{~cm}$ long, $12.7 \mathrm{~cm}$ diameter warm (although radiatively cooled by the inner liquid nitrogen shield) bore passed through a set of main windings which generated the $7 \mathrm{~T}$ high-field region at the entrance to the magnet as well as the $1 \mathrm{~T}$ precision gradient field region (see Fig. 1) when energized to a maximum current of 96.45A. Current was supplied to this main coil by an AMI 12100 power supply controlled by an AMI 420 power supply programmer. Ten superconducting shim coils spaced every $\sim 5.1 \mathrm{~cm}$ and centered on the $1 \mathrm{~T}$ region provided tailoring of the field in the precision gradient region and were controlled by an Oxford ISS 10 shim controller.

The spin flipper was installed in the AFP magnet around a quartz tube coated inside with DLC which provided a high material potential surface for UCN while not screening the rf field, ${ }^{26}$ and rf power was supplied to the spin flipper from an Amplifier Research broadband rf amplifier (AR150A) fed by a Wavetek precision frequency synthesizer (model\# 5135A). Cooling water was provided by a Neslab chiller outputting $10 \mathrm{gph}$ at $20 \mathrm{psi}$, which kept the equilibrium coil tempera- ture stable to within $\pm 1{ }^{\circ} \mathrm{C}$ for input powers up to $100 \mathrm{~W}$, the highest power utilized in these tests. (Based on prototype testing at higher powers using an additional amplifier, it is expected that stable operation should be possible for at least $500 \mathrm{~W}$ of input power.) Coil temperature, measured using the resistance temperature detector, was maintained at $\sim 12{ }^{\circ} \mathrm{C}$. The cooling water used was filtered tap water, and there were no observable changes in system performance associated with the presence of the cooling water. Forward and reflected rf power were monitored using a Bird Wattcher rf monitor/alarm (model\# 3170A), and at the ideal driving frequency the reflected power was $<1 \%$ of the forward power (with no changes to the matching capacitor settings determined on the bench).

Prior to installing the spin flipper in the AFP magnet (but with the DLC guide installed through the cavity), a $1 \mathrm{~cm}$ diameter sensor loop, placed at the center of the cavity and oriented to create a maximum signal when connected to the $50 \Omega$-terminated input of an oscilloscope, was used to calibrate the spin flipper's internal sensor loop (mounted flush against the outside of one of the rungs $90^{\circ}$ from the drive connections and just above the tuning ring). These measurements are shown in Fig. 8 and indicate that the magnitude of the rotating $B_{1}$ field produced in the cavity (half the total field by Eq. (14)) depends on the power reaching the cavity as approximately

$$
B_{1}=\frac{1}{2} B_{\mathrm{rf}}=\left(0.15 \pm 0.01 \mathrm{G} / \mathrm{W}^{0.52}\right) P^{(0.52 \pm 0.02)},
$$

and that the internal sensor loop has a linear response. In particular,

$$
B_{1}=(0.047 \pm 0.001 \mathrm{G} / \mathrm{V}) V_{\mathrm{pk} 2 \mathrm{pk}}-(0.0003 \pm 0.01 \mathrm{G}),
$$

where $V_{\mathrm{pk} 2 \mathrm{pk}}$ is the peak-to-peak voltage recorded on a $50 \Omega$ terminated oscilloscope channel for the internal sensor loop. In situ measurement of the rf field profile was not possible at the time of these measurements since it was feared that the probe would damage the DLC coating on the UCN guides, but was later performed with the quartz DLC flipper guide coupled to the $\mathrm{Cu}$ UCN guides used in UCNA. The result of this subsequent measurement is shown in Fig. 1.
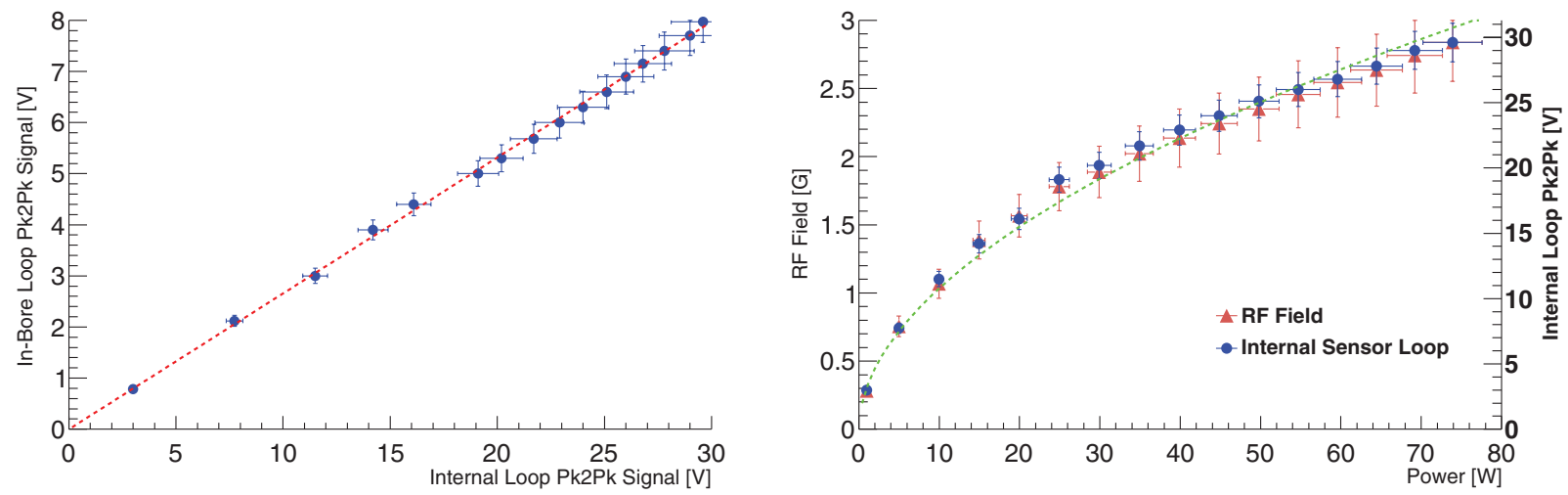

FIG. 8. (Left) Comparison of the spin flipper internal pickup loop to an in-bore loop prior to installation. (Right) Relationship between the rf field in the cavity calculated using an in-bore pickup loop signal and the input power, with the corresponding internal pickup loop signal voltages also shown (right axis, circles). Dashed curves are fits to the data (see text). 


\section{B. Crossed polarizer analyzer experimental geometry}

UCN produced in $5 \mathrm{~K} \mathrm{SD}_{2}$ via downscattering of spallation neutrons pre-moderated by $\sim 20 \mathrm{~K}$ polyethylene ${ }^{25}$ were horizontally extracted, after rising about $1 \mathrm{~m}$ against gravity, and then transported through two separated $45^{\circ}$ bends and $\sim 5 \mathrm{~m}$ of electropolished stainless steel (EPS) guide $(\sim 10 \mathrm{~cm}$ diameter) to a gate valve outside the radiation shielding. Just prior to the gate valve there was a small $\sim 0.635 \mathrm{~cm}$ diameter hole in the bottom of the guide which was coupled to $a^{3} \mathrm{He}$ UCN detector ${ }^{27}$ (the monitor detector) through a drop of about $1 \mathrm{~m}$, allowing the UCN density at that point in the system to be monitored. The guide emerging from the gate valve had a diameter of $7.6 \mathrm{~cm}$ and transported UCN $1.71 \mathrm{~m}$ through a $\sim 127 \mu \mathrm{m}$ thick Al safety foil (isolating the vacuum system containing the $\mathrm{SD}_{2}$ from the guides comprising the experiment) and then to an EPS $60^{\circ}$ elbow connected to a shutter. The purpose of the shutter was both to provide timeof-flight information and to reduce the number of multiplepass UCN trajectories in the downstream guides. The latter was accomplished by affixing three layers of $0.254 \mathrm{~mm}$ thick polymethylpentene (TPX), a good neutron absorber, to the downstream side of the shutter. Following the shutter, another $\sim 34.2 \mathrm{~cm}$ of $7.6 \mathrm{~cm}$ diameter EPS guide was followed by a transition to $7 \mathrm{~cm}$ diameter EPS guide and then to $7 \mathrm{~cm}$ diameter DLC-coated quartz guide. The DLC section, formed by five coupled guides including the one installed through the spin flipper, ran for about $3.9 \mathrm{~m}$, passing first through the AFP magnet and then through a meter-long superconducting solenoidal magnet called the pre-polarizer magnet (PPM), which for UCNA is normally positioned upstream of the AFP magnet around the $\mathrm{Al}$ safety foil to accelerate one spin state through the foil. Fig. 1 provides an overview of the experimental setup with the field profile created by the two magnets indicated. At the downstream exit of the PPM, the final DLC guide was coupled to a $7.6 \mathrm{~cm}$ diameter EPS $30.4 \mathrm{~cm}$ radius $90^{\circ}$ elbow which was connected through a $1 \mathrm{~m}$ drop to a second ${ }^{3} \mathrm{He} \mathrm{UCN}$ detector (the main detector). This configuration contained the requisite elements to form a crossed polarizer analyzer, with the AFP magnet plus spin flipper serving as the crossed polarizer and the PPM functioning as the analyzer.

\section{UCN longitudinal spectrum measurement}

Neutrons for which the $7 \mathrm{~T}$ longitudinal field of the AFP magnet presents a repulsive potential barrier (low-field seeking UCN) but which have a longitudinal velocity high enough that no classical turning points due to the potential exist will be able to pass through the polarizing field. If those neutrons have their spin reversed by the spin flipper, they will see the analyzing field as an attractive potential and therefore not be stopped (analyzed) by the PPM regardless of its maximum field. Since any population of this type creates a leakage signal through the crossed polarizer analyzer, it is important to measure directly the size of this population. In other words, a measurement of the polarizing efficiency (or polarizing power) is important to properly interpret the results of a crossed polarizer analyzer measurement. Since the spin flip efficiency in an adiabatic fast passage spin flipper depends on longitudinal speed, a measurement of the longitudinal UCN spectrum is also important for understanding the action of the spin flipper. For UCN, which are kinematically polarized, these two measurements are equivalent.

UCN spectra are often obtained using gravity as a retarding potential analyzer. When working with polarized UCN, however, where a polarizing magnetic field is invariably present, use may be made of the polarizing field itself as a retarding potential. In that case, when transport effects are negligible and the incident UCN population is unpolarized, the transmission as a function of polarizing magnet field (with the analyzer field held at $0 \mathrm{~T}$ and the spin flipper off) should behave as

$$
T\left(B_{\text {afp }}\right)=\frac{\frac{1}{2} \int_{0}^{\infty} \rho_{z}(\eta) \mathrm{d} \eta+\frac{1}{2} \int_{\hat{v}(B)}^{\infty} \rho_{z}(\eta) \mathrm{d} \eta}{\frac{1}{2} \int_{0}^{\infty} \rho_{z}(\eta) \mathrm{d} \eta+\frac{1}{2} \int_{0}^{\infty} \rho_{z}(\eta) \mathrm{d} \eta},
$$

where $\rho_{z}$ represents the probability density for finding a UCN with longitudinal speed $v$, and $\hat{v}(B)=\varkappa \sqrt{B}(\varkappa$ $=3.387 \mathrm{~m} / \mathrm{sT}^{1 / 2}$ ) gives the largest neutron speed which results in a classical turning point for a low-field seeking UCN. The first term of Eq. (20) represents a background comprised of UCN that see the polarizer field as an attractive potential (high-field seeking $\mathrm{UCN}$ ) and which is therefore present for all values of the polarizing field, while the second term represents the low-field seeking UCN, which are attenuated as the polarizing field is increased. Taking the derivative of Eq. (20) gives

$$
\rho_{z}(v) \propto-\left.v \frac{\mathrm{d} T}{\mathrm{~d} B}\right|_{B=(v / \varkappa)^{2}}
$$

so that forming the differential spectrum from a polarizing field scan is expected to provide direct information about the longitudinal velocity distribution and hence the polarization of the UCN population reaching the spin flipper. Specifically, a non-zero value of the differential spectrum at $v_{z}=\varkappa \sqrt{B_{\max }}$ $(8.96 \mathrm{~m} / \mathrm{s}$ for a $7 \mathrm{~T}$ polarizing field) would be evidence of a polarization less than $100 \%$, which would show up as leakage through the crossed polarizer analyzer.

A polarizing magnet field scan was performed with the proton beam pulsed at a rate of $1 \mathrm{~Hz}$ while the gate valve and shutter were opened for $1 \mathrm{~s}$ out of every $10 \mathrm{~s}$. Fig. 9 shows characteristic timing spectra for the main and monitor detectors, and indicates that the $10 \mathrm{~s}$ cycle time was sufficiently long to allow the system to empty prior to the next cycle. With the PPM held at $0 \mathrm{~T}$ and the spin flipper off, total counts in the main and monitor detectors over a number of consecutive $10 \mathrm{~s}$ cycles were recorded as a function of the maximum AFP magnet field. The background in the main detector was generally measured twice in an 8-h period with the proton beam on and the shutter cycling but the gate valve closed. (Proton-beam-on backgrounds were not obtainable for the monitor detector since it was located upstream of the gate valve. Typical rates in the monitor detector were $\sim 17 \mathrm{~Hz}$, however, significantly larger than the typical $\sim 60 \mathrm{mHz}$ background rate for the ${ }^{3} \mathrm{He}$ detectors, and pulse height spectra were examined for any appearance of noise.) At each AFP field value the background-subtracted main detector counts were normalized by the monitor counts, and transmissions 

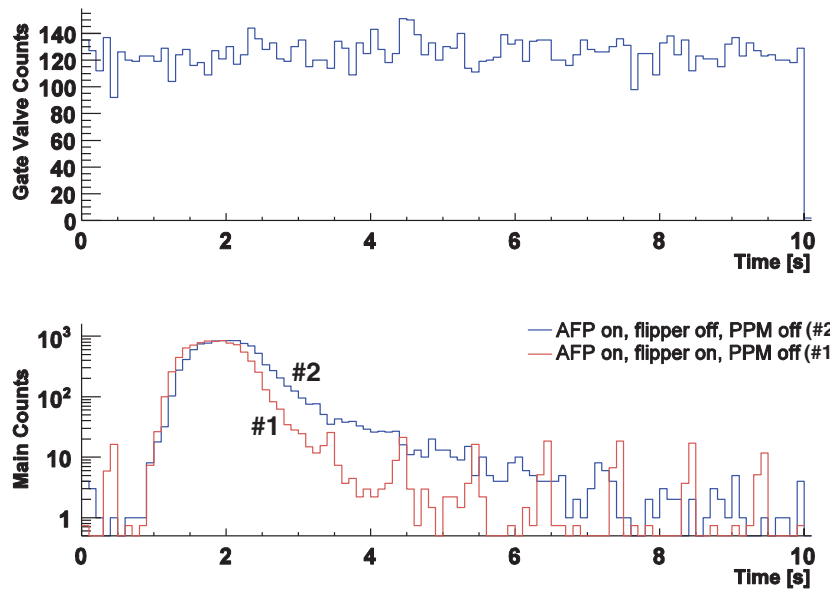

FIG. 9. Typical raw timing spectrum representing 128 shutter cycles summed together for the monitor detector (top) and the main detector with the AFP magnet on, flipper off, and PPM off (bottom; blue, \#2). The periodic spikes in the lower spectra are beam induced background. The flipper on (red, \#1) spectrum, whose peak has been normalized to the flipper off spectrum, leads the flipper off spectrum due to the $120 \mathrm{neV}$ total energy boost UCN acquire when spin flipped in $1 \mathrm{~T}$.

calculated by dividing the normalized main detector counts by the value obtained with the AFP magnet held at zero field. Fig. 10 shows the results along with the calculated differential spectrum.

\section{D. $B_{0}$ tuning}

Prior to performing crossed polarizer measurements, the field in the $1 \mathrm{~T}$ region of the AFP magnet needed to be matched with the spin flipper drive frequency to ensure that the resonance condition was met in the rf cavity. Since
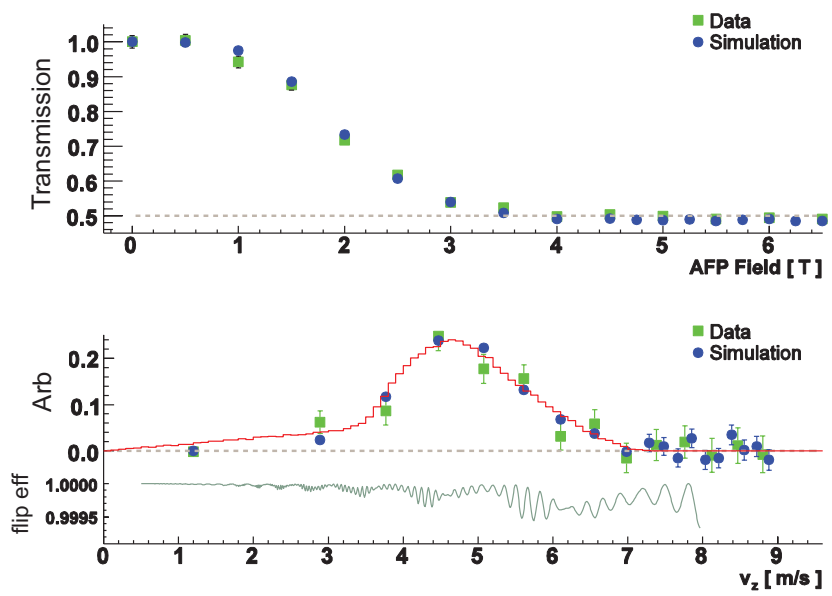

FIG. 10. (Top) AFP magnet spectrum scan compared to simulation. (Bottom) Longitudinal velocity spectra resulting from the actual and simulated magnet scans from the upper plot compared with the actual longitudinal velocity spectrum at the location of the maximum field point (but with the field off) predicted by the simulation (red line). The linear tail at low longitudinal speeds (cf. Fig. 15) is due to the presence of the Al safety foil downstream of the gate valve. Also plotted for comparison is the spin flip efficiency as a function of longitudinal velocity computed by integrating Eq. (5) using the measured rf field profile and the measured $B_{0}$ field combined with the measured individual shim coil responses scaled for the $0.1 \mathrm{G} / \mathrm{cm}$ shim coil current settings.

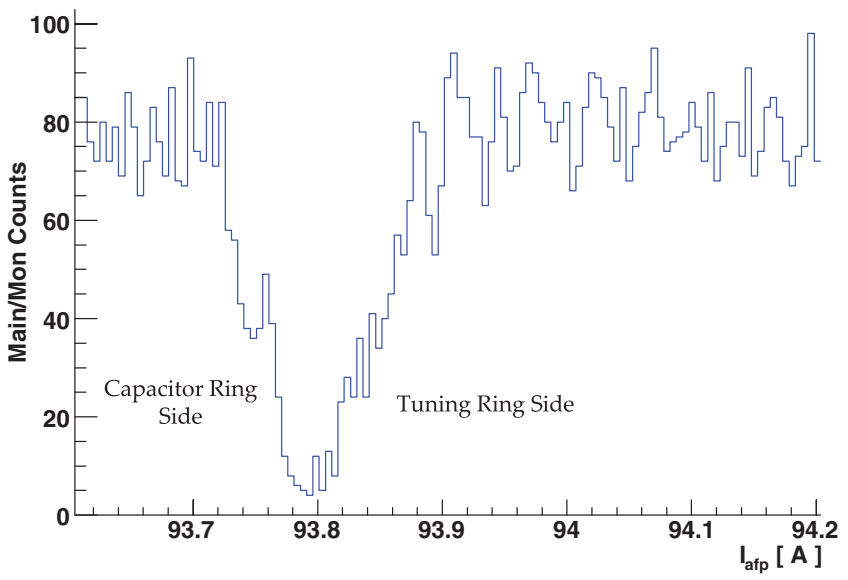

FIG. 11. An AFP magnet tuning current scan (shim coils off) with no Cu foil in the PPM high-field region. As the current increases the resonance point moves through the capacitor ring and towards the tuning ring.

the AFP magnet did not possess a power supply capable of simultaneously addressing all ten of its shim coils, this $B_{0}$ tuning was performed in two steps. First, with the PPM at $6 \mathrm{~T}$, the spin flipper driven at the frequency $f$ which produced a minimum reflected power, and the shims coils held at $0 \mathrm{~A}$, the AFP magnet current was continuously ramped while recording rates in the main and monitor detectors. During this current tuning scan, as the $B_{0}$ field in the rf cavity increased, the physical location of the resonance point where $\gamma B_{0}$ $=2 \pi f$ moved at a rate dependent on both the speed at which the current was changed and the $B_{0}$ field gradient through the cavity, producing the curve shown in Fig. 11. Fine tuning of the spin flipper system was then accomplished by setting the AFP magnet current to coincide with the minimum value from the current scan, setting the shim coils to achieve a desired field gradient through the rf cavity, and performing a frequency scan where transmission through the system (calculated as described above) was measured as a function of birdcage driving frequency. The results of these scans for various $B_{0}$-field gradients and input powers are shown in Fig. 12. As in the case of the current scan, changing the frequency changed the location of the resonance point in the rf cavity (albeit in the opposite direction), so that the shape of a frequency scan is determined by a folding together of the $B_{0^{-}}$and $B_{1}$-field profiles. Notice, for instance, that smaller field gradients correspond to wider flat regions in the frequency scans. The operation frequency for the spin flipper at the selected AFP magnet current was generally chosen to be the one at the minimum of a frequency scan performed at $5 \mathrm{~W}$ of input power as depicted in Fig. 12. (Frequency scans at $5 \mathrm{~W}$ were utilized for tuning since at higher input powers it was increasingly difficult to resolve any structure in the flat bottom of the scan.) In the event that the selected frequency resulted in a reflected power greater than $1 \%$ of the input power, the AFP magnet current could be adjusted to shift the frequency closer to the reflected power minimum.

\section{E. Crossed polarizer analyzer measurements}

The primary dependence to study with the crossed polarizer analyzer is the transmission through the system as a 

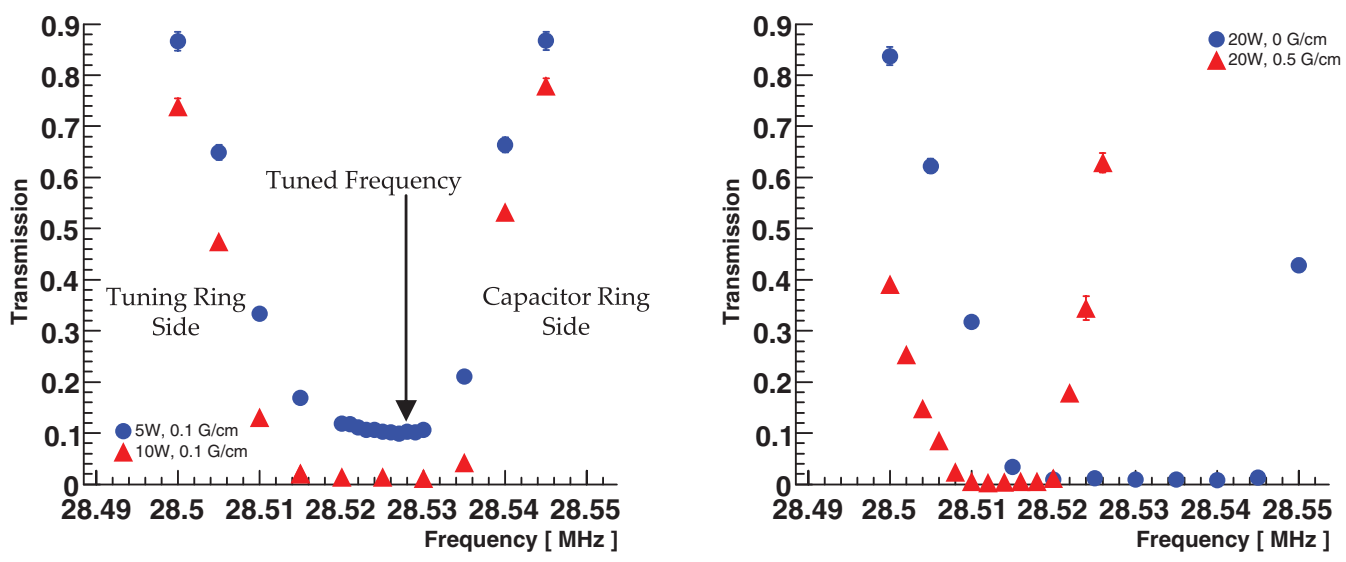

FIG. 12. Frequency scans showing the effect of changing the $B_{0}$-field gradient (by altering shim coil settings) and the effect of changing the input power to the birdcage coil. As the frequency is increased, the resonance point moves through the tuning ring and towards the capacitor ring. A typical tuned flipper operation frequency was $28.527 \mathrm{MHz}$ for an AFP magnet current of $93.76 \mathrm{~A}$, which corresponded to a maximum polarizing field of $6.8 \mathrm{~T}$.

function of input power; since spin flip efficiency should scale with rf field magnitude (and hence spin flipper input power) according to Eq. (11) while typical sources of leakage should not, such a measurement provides a way to separate these effects. It is nevertheless important to minimize leakage, which means that not only must the polarizing efficiency be high, but the polarizing and analyzing powers must be equivalent. This equivalence requires (assuming no prior information about the UCN spectrum) that the PPM produce a field of 9T. The necessity of having the analyzer field be larger than the polarizer field follows from the fact that when a high-field seeking UCN has its spin reversed in the $1 \mathrm{~T}$ region of the AFP magnet, the potential it sees changes from attractive to repulsive. Since the kinetic energy remains constant during the absorption of a photon from the rf field in the cavity, the total energy of the UCN must undergo a change $\Delta E=+120 \mathrm{neV}$. This means that the potential barrier presented to spin-flipped $\mathrm{UCN}$ by the analyzer must be $120 \mathrm{neV}$ greater than the barrier presented by the polarizing magnet if the analyzing and polarizing powers are to be the same. Recalling that the magnetic potential for neutrons is $60 \mathrm{neV} / \mathrm{T}$, this requires that the analyzer field be $2 \mathrm{~T}$ higher than the polarizing field for an ideal crossed polarizer analyzer configuration. Analyzer fields lower than this have the potential to allow some fraction of the spin-flipped UCN spectrum to pass, thus creating a source of leakage.

Unfortunately, the PPM is capable of a maximum field of only $7 \mathrm{~T}$, and safety concerns (primarily uncertainty about the effects of a PPM quench while in close proximity to the AFP magnet) limited the maximum PPM field for these measurements to $6 \mathrm{~T}$. In order to combat this significant lack of analyzing power, a $0.0127 \mathrm{~mm}$ thick $\mathrm{Cu}$-coated Kapton foil (diameter $\sim 7 \mathrm{~cm}$ ) was epoxied to a $1.1 \mathrm{~cm}$ thick Teflon ring $(0.4 \mathrm{~cm}$ wall, $6.3 \mathrm{~cm} \mathrm{OD})$, which was installed at the center of the PPM high-field region. (Two pieces of $\mathrm{BeCu} 0.0762 \mathrm{~mm}$ diameter wire $\sim 53 \mathrm{~cm}$ long were attached to the downstream side of the Teflon ring to facilitate removal of the foil.) Since $\mathrm{Cu}$ has a material potential of around $168 \mathrm{neV}$, this additional (spin independent) potential barrier nominally raised the total analyzing potential to $\sim 520 \mathrm{neV}$, just $20 \mathrm{neV}$ below the potential presented by a $9 \mathrm{~T}$ field.
In order to assess directly the analyzing power of the PPM field plus $\mathrm{Cu}$ foil, analyzer field scans were performed with and without the foil in place. These measurements (shown in Fig. 13) were essentially the same as the spectrum measurement described in Sec. III C, except that the AFP magnet current was held constant (at the value determined during spin flipper tuning) and the spin flipper was on while the PPM current was varied. Just as a non-zero slope at maximum polarizing field in the AFP magnet scan is indicative of an imperfect polarizer, a non-zero slope in the crossed polarizer analyzer PPM scan result at maximum analyzing field indicates imperfect analyzing power (so that the no-foil scan shown in Fig. 13 clearly demonstrates the importance of the
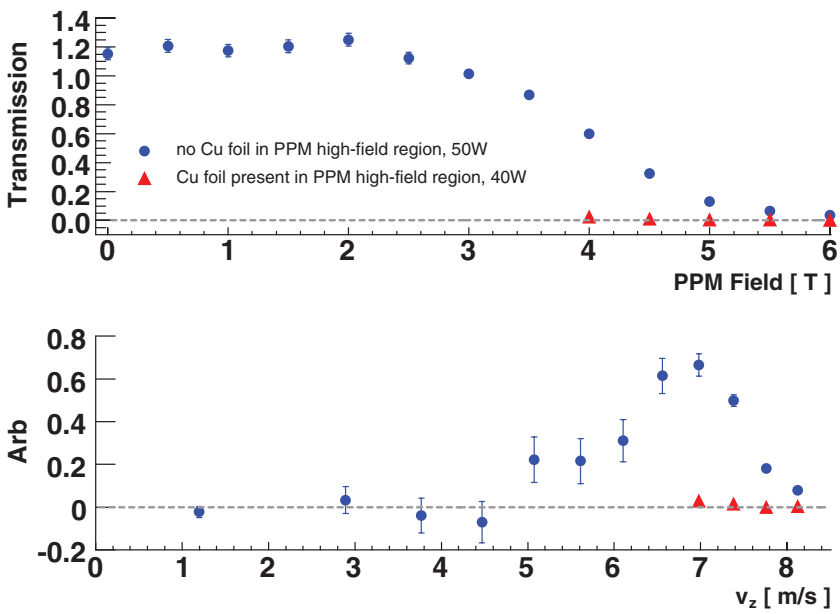

FIG. 13. (Top) Retarding potential scans using the analyzer (PPM) field with the spin flipper activated. Transmissions are calculated relative to the rate with the analyzer field at $0 \mathrm{~T}$ and the flipper off, which results in transmissions greater than one due to velocity dependent transmission effects. The circles show a scan without the $\mathrm{Cu}$ foil in the PPM high-field region and were performed at $50 \mathrm{~W}$ with the shutter always open, while the triangles show a scan with the $\mathrm{Cu}$ foil in the PPM high-field region and were performed at $40 \mathrm{~W}$ with the shutter operating normally. (Bottom) Longitudinal velocity spectra resulting from the two scans depicted above. Comparing these spectra to the spectrum in Fig. 10, the $120 \mathrm{neV}$ boost caused by the action of the spin flipper is clearly discernible. The importance of the $\mathrm{Cu}$ foil is also readily apparent in the inability of the PPM field alone to fully analyze the spin-flipped spectrum. 


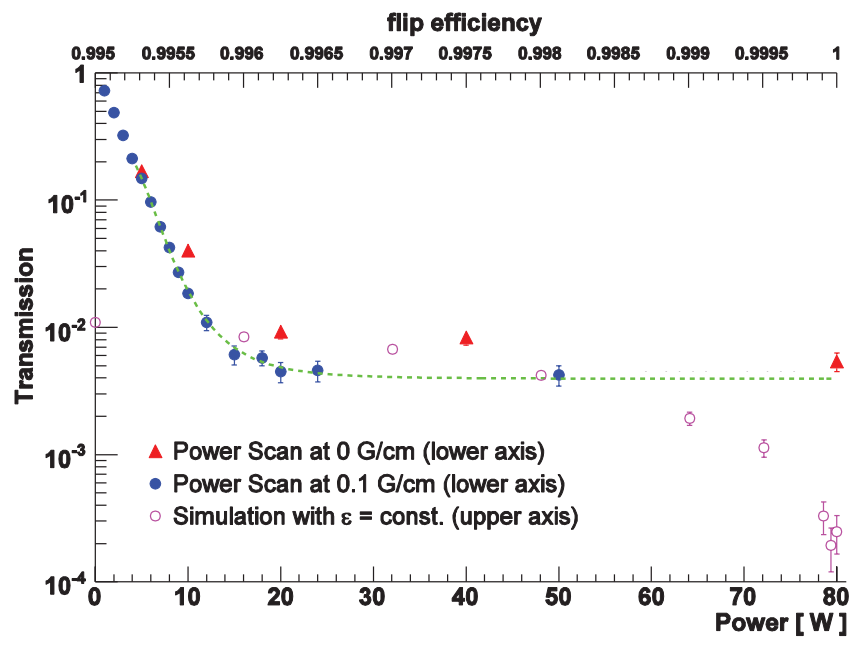

FIG. 14. Comparison of power scans with shim coils set for $0.1 \mathrm{G} / \mathrm{cm}$ and $0 \mathrm{G} / \mathrm{cm}$. Note that an arbitrarily small gradient does not result in an enhanced spin flip efficiency. Fitting with a model $\frac{A}{B+P v}+C$, which has the form of Eq. (11) plus a constant, results in $C=0.0039 \pm 0.0004$, giving a lower limit for the average spin flip efficiency of $\bar{\epsilon}>99.6 \%$. Monte Carlo simulations with a constant spin flip efficiency produce the open circles (associated with the upper scale). They include spectral and transport effects and imply an actual average spin flip efficiency of $\sim 99.8 \%$, but do not include velocity dependence of the flip efficiency.

foil for subsequent measurements). Finally, with the foil in place, measurements of the transmission through the system as a function of flipper driving power (power scans) were performed as depicted in Fig. 14.

\section{RESULTS AND CONCLUSIONS}

The spin flip efficiency in a spin flipper of the type described here is expected to depend on the longitudinal speed of the flipped UCN (cf. Eq. (11)), so that what is actually measured by the transmission $T$ through an ideal crossed polarizer analyzer is the average flip efficiency

$$
\bar{\epsilon}\left(B_{1}\right)=\int_{0}^{\infty} \rho_{z}\left(v_{z}\right) \epsilon\left(v_{z}, B_{1}\right) \mathrm{d} v_{z}=1-T\left(B_{1}\right) .
$$

In the case of a realizable crossed polarizer analyzer, there are potential sources of leakage as well as transport effects so that the measured transmission is connected to the spin flip efficiency via

$$
\begin{aligned}
T\left(B_{1}\right)= & \int_{0}^{\infty} \rho_{z}\left(v_{z}\right) T\left(v_{z}, B_{1}\right) \mathrm{d} v_{z} \\
= & \frac{1}{2} \int_{0}^{\infty} \rho_{z}\left(v_{z}\right) \Theta^{+}\left(v_{z}, B_{1}\right) \\
& \times\left[1-\epsilon\left(v_{z}, B_{1}\right)+\xi^{+}\left(v_{z}\right)+\chi^{+}\left(v_{z}, B_{1}\right)\right] \mathrm{d} v_{z} \\
& +\frac{1}{2} \int_{0}^{\infty} \rho_{z}\left(v_{z}\right) \Theta^{-}\left(v_{z}, B_{1}\right) \\
& \times\left[1-\epsilon\left(v_{z}, B_{1}\right)+\xi^{-}\left(v_{z}\right)+\chi^{-}\left(v_{z}, B_{1}\right)\right] \mathrm{d} v_{z}
\end{aligned}
$$

where \pm indicates the incident spin state, $\xi$ represents $B_{1}$ field independent sources of leakage, $\chi$ represents $B_{1}$-field dependent sources of leakage, and $\Theta$ incorporates transport corrections (dependence on $\nabla_{z} B_{0}$ has been suppressed and the incident UCN are assumed unpolarized). Contributions to $\xi$ include imperfect initial polarization, insufficient analyzing power, and UCN depolarization (due to material interactions with the guides, Majorana spin flips, or failures in adiabatic transport due to static field gradients), while a non-zero $\chi$ may be caused by the existence of multiple positions in the system which satisfy the resonance condition or UCN depolarization due to wall reflections in the presence of the $B_{1}$ field. The transport corrections $\Theta$ include effects such as multiple passes through the spin flipper and velocity dependent transmission (caused by the fact that UCN with higher longitudinal speeds tend to undergo fewer bounces and therefore a reduced loss per meter of guide traversed).

In order to unfold the contribution of transport effects from the results of Fig. 14 as well as to explore the magnitude of possible contributions to $\xi$ and $\chi$, a Monte Carlo UCN transport $\operatorname{code}^{9}$ was employed which provided a model of the entire experimental geometry seen by UCN (including the $\mathrm{SD}_{2}$ source). For the purpose of this analysis, UCN were assumed to have classical ballistic trajectories between bounces determined by both gravity and, where appropriate, longitudinal magnetic forces due to gradients calculated from linear approximations of measured longitudinal fields (see Fig. 1). Wall interactions were modeled by a constant probability of specular scatter (with purely diffuse non-specular scattering, distributed as $\rho(\theta, \phi) \sim \sin \theta \cos \theta$ into $2 \pi)$, a constant probability of depolarization per bounce, and a probability of loss per bounce $\lambda$ modified by an energy dependent factor ${ }^{2}$

$$
P_{\mathrm{loss}}(E)=\left\{\begin{array}{l}
2 \lambda \sqrt{\frac{E_{\perp}}{E_{\mathrm{F}}-E_{\perp}}} \text { for } E_{\perp}<\frac{E_{\mathrm{F}}}{4 \lambda^{2}+1} \\
1 \text { for } E_{\perp} \geq \frac{E_{\mathrm{F}}}{4 \lambda^{2}+1}
\end{array}\right.
$$

where $E_{\perp}$ represents the kinetic energy associated with the velocity component perpendicular to the surface at the location of the bounce. The $\mathrm{Cu}$ foil was modeled as presenting a fixed material potential to an incident neutron, but with a specified probability (the foil leakage fraction) for the neutron to miss the foil entirely by passing through a pinhole in the foil or a gap between the foil and the neutron guide. Each UCN was created at the gate valve with an angular distribution $\rho(\theta, \phi)$ $\sim \sin \theta \cos \theta$ into the downstream half-space with a (normalized) distribution of speeds (see Fig. 15)

$$
\rho(v) \sim \begin{cases}v^{n} & \left(0 \leq v \leq v_{\mathrm{pk}}\right) \\ \left(\frac{v_{\mathrm{pk}}^{n}}{v_{\mathrm{cut}}-v_{\mathrm{pk}}}\right)\left(v_{\mathrm{cut}}-v\right) & \left(v_{\mathrm{pk}} \leq v \leq v_{\mathrm{cut}}\right),\end{cases}
$$

whose form was chosen based on results from a previous study of the UCN spectrum at the gate valve which utilized the PPM as a retarding potential analyzer. ${ }^{28}$ Creation of the UCN was uniformly distributed in time over an interval of $1.6 \mathrm{~s}$ with the gate valve opening at $t=0 \mathrm{~s}$ and closing at $t=2 \mathrm{~s}$ and the shutter opening at $t=0.5 \mathrm{~s}$ and closing at $t=1.5 \mathrm{~s}$. Communication with the $\mathrm{SD}_{2}$ source and guides upstream of the gate valve was possible, but all UCN upstream of the shutter were killed off after the shutter was closed in order to save computational time. 


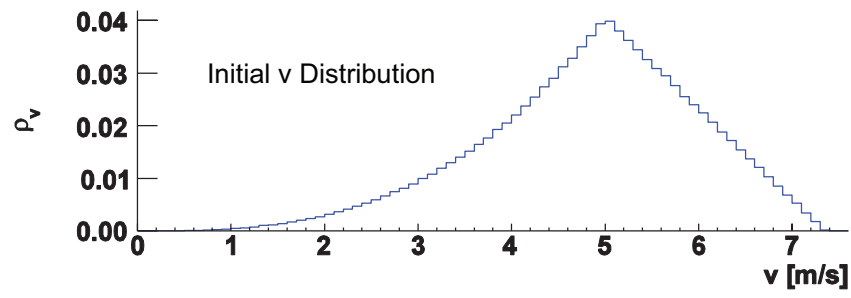

FIG. 15. Initial speed distribution for the Monte Carlo calculations generated by Eq. (25) with $n=2.85, v_{\mathrm{pk}}=5.0 \mathrm{~m} / \mathrm{s}$, and $v_{\text {cut }}=7.35 \mathrm{~m} / \mathrm{s}$.

This Monte Carlo model was first applied to the polarizer spectrum scan of Sec. III C by fixing model parameters for all EPS surfaces (specularity $(\mathrm{spec})=0.96$, depolarization-perbounce $(\mathrm{dpb})=1.0 \times 10^{-4}$, and loss-per-bounce $(\mathrm{lpb})=1.0$ $\times 10^{-5}$, where lpb on EPS is typically higher, on the order of $2 \times 10^{-4}$, but since this value had no discernible effect on the observables it was set artificially low to enhance the simulation statistics) and then individually varying parameters in the set $\left\{n, v_{\mathrm{pk}}, v_{\text {cut }}\right.$, DLC spec, DLC lpb, DLC material potential $\}$ to minimize $\chi^{2}$ between model predictions and data. Model transmissions were calculated based on counts reaching the main detector (assuming that UCN which passed through the Al detector foil were detected with $100 \%$ efficiency) normalized by the number of UCN generated in the simulation, and quadratic fits to the $\chi^{2}(p)$ functions (with $p$ the relevant parameter) were used to establish a central value corresponding to the $\chi^{2}$ minimum and error intervals corresponding the points where $\chi^{2}=\left(\chi^{2}\right)_{\min }+1$. The results are shown in the top block of Table I and in Fig. 10.

In order to establish values for the material potential and leakage fraction of the $\mathrm{Cu}$ foil, the Monte Carlo model was set for a constant spin flip efficiency of 0.999 and used to calculate transmissions for conditions corresponding to the analyzer magnet (PPM) scan performed with the foil in place. Since the differential spectrum carries information only about the fraction of UCN which are actually analyzed and so de-

TABLE I. (Upper block) Model parameter values determined by the spectrum scan measurements of Sec. III C by individually varying the indicated parameter in order to determine a minimum $\chi^{2}$ and an error interval. The error intervals indicate the range of parameter values for which the model agrees with the experimental data at the $1 \sigma$ level; hence larger errors indicate reduced sensitivity to the central parameter value. (Lower block) Results of minimizing $\chi^{2}$ between the differential spectrum of the analyzer field scan (with $\mathrm{Cu}$ foil) from Sec. III $\mathrm{E}$ and simulation for simultaneous variations in the material potential and leakage fraction of the $\mathrm{Cu}$ foil. All variations were performed with the other parameters fixed at the values recorded in the right column.

\begin{tabular}{lccc}
\hline \hline Parameter & Estimated value & $\left(\chi^{2} / \nu\right)_{\min }$ & Base value \\
\hline $\mathrm{n}$ & $1.25 \pm 0.87$ & 0.80 & 2.85 \\
$v_{\text {pk }}(\mathrm{m} / \mathrm{s})$ & $4.55 \pm 0.18$ & 0.69 & 5.0 \\
$v_{\text {cut }}(\mathrm{m} / \mathrm{s})$ & $7.32 \pm 0.11$ & 1.02 & 7.35 \\
$\mathrm{DLC}$ potential $(\mathrm{neV})$ & $200 \pm 80$ & 0.98 & 150 \\
$\mathrm{lpb}$ & $(1.35 \pm 0.63) \times 10^{-3}$ & 0.70 & $5.0 \times 10^{-5}$ \\
$\mathrm{spec}$ & $0.99997_{-0.004}^{+0.0003}$ & 0.5 & 0.990 \\
$\mathrm{dpb}$ & $\ldots$ & $\ldots$ & $2.0 \times 10^{-6}$ \\
Foil potential (neV) & $135_{-6}^{+265}$ & 1.4 & \\
Foil leak & $0.021 \pm 0.005$ & 1.4 & \\
\hline \hline
\end{tabular}

pends strongly on the analyzing power-i.e., the foil potential and leakage fraction-and not on the spin flipper efficiency, it is the slope of the analyzer magnet scan close to the maximum analyzer field which must be compared to model results in order to constrain the $\mathrm{Cu}$ foil potential and leakage fraction. So, $\chi^{2}$ between the differential spectrum calculated from the data and the differential spectrum calculated from the Monte Carlo model transmissions was determined for a range of foil leakage fractions, with each such parameter scan performed over a range of values for the foil potential. Taking $\chi^{2}(u, l)$ (where $u$ is the foil potential and $l$ is the leakage fraction) to have a quadratic form, best parameter values were identified as those corresponding to the $\chi^{2}$ global minimum, and parameter errors were determined by estimating the corresponding $\chi^{2}=\left(\chi^{2}\right)_{\min }+1$ error ellipse. The resulting values are shown in Table I. The effect of the constant flipper efficiency used in the determination of these parameters was investigated by repeating a subset of the calculations with a constant spin flip efficiency of 0.995 , and $\chi^{2}(u, l)$ computed by comparing differential spectra was indeed found to be insensitive to the constant efficiency used in the model. As can be seen in Table I, the foil potential is well-constrained for values smaller than $135 \mathrm{neV}$, but is essentially unconstrained for larger values. This is to be expected since once the foil material potential is great enough to fully analyze the entire incident spectrum, increases in that potential should have no effect on the behavior of the system. The upper limit for the foil material potential was therefore selected to be $400 \mathrm{neV}$, corresponding to an energy just beyond the UCN range.

After constraining the Monte Carlo model using the magnet scans (which effectively determines the polarizing and analyzing power of the system), the remaining unknown parameters were the depolarization probability per bounce on DLC, the UCN absorption coefficient on the downstream side of the shutter, and also the exact form for the spin flip efficiency (imagined here to incorporate all $B_{1}$-field dependent effects), which must be taken as unknown despite, for example, Eq. (5) since even with a perfectly determined $B_{1}$ profile, the theory does not incorporate the physics of bounces in the $\mathrm{rf}$ region. Since assuming a constant spin flip efficiency is not commensurate with the results of UCNA polarimetry measurements, ${ }^{9}$ a quadratic model for the longitudinal velocity dependence of the flip efficiency (cf. Eq. (11)) was used, which introduces a single additional parameter $c_{2}$ defined by

$$
\epsilon(v)=1+c_{2} v^{2},
$$

where $v$ is a small dimensionless parameter which depends on the longitudinal velocity (and is defined in terms of characteristic $B_{1}$ and $\nabla_{z} B_{0}$ values using Eq. (11)), here taken to be

$$
v \equiv \frac{v_{z}}{\sqrt{\frac{\gamma^{2} B_{1}^{4}}{\left(\nabla_{z} B_{0}\right)^{2}}}}=\frac{100}{\sqrt{2.6244 \times 10^{8}}} v_{z}
$$

for $v_{z}$ in $\mathrm{m} / \mathrm{s}$. Since no specific experimental input about the depolarization per bounce on the DLC guides used in this measurement was available, a value of $c_{2}$ which put the transmission predicted by the model in agreement with the empirically determined asymptotic transmission from Fig. 14 for 


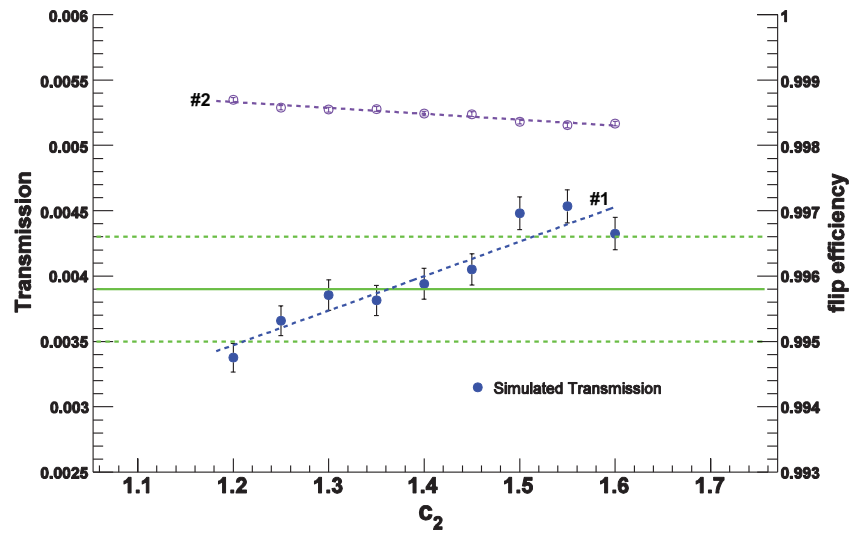

FIG. 16. Simulated transmission as a function of $c_{2}$ compared to the empirically determined asymptotic value of the transmission. The dashed blue line (\#1) is a linear fit to the simulation results and the dashed purple line (\#2) is a linear fit to the average spin flip efficiency (left axis) calculated directly in each simulation. The horizontal (green) lines show the empirically determined transmission and its $\pm 1 \sigma$ error envelope.

the case of no material depolarization and $100 \%$ absorption on the shutter was obtained by calculating the transmission through the system as a function of $c_{2}$, with the other parameters set to their previously determined best values. The results of these calculations (shown in Fig. 16) were fit with a line, whose intersections with the data central value and $\pm 1 \sigma$ values determined $c_{2}$ to be $1.36 \pm 0.2$.

In order to incorporate the model parameter uncertainties into the error on $c_{2}$, transmissions through the system were calculated with $c_{2}=1.36$ and each parameter varied to include the range indicated in Table I. The error induced in the determination of $c_{2}$ was estimated by assuming the slope of transmission versus $c_{2}$ remained constant and calculating a new intersection point with the data central value, which gave an induced variation in $c_{2}$. The results of these calculations are shown in Table II. Since these various errors are correlated, they were directly summed with the above error to give $c_{2}=1.36_{-0.48}^{+0.34}$. Finally, by directly evaluating the average spin flip efficiency for each of the simulations represented in Fig. 16 and fitting the result with a line (shown in the figure), the average spin flip efficiency corresponding to a particular value of $c_{2}$ was determined. Using this relationship, an average spin flip efficiency of $\bar{\epsilon}=0.9985 \pm 0.0004$ was obtained, corresponding to the case of no material de-

TABLE II. Errors induced in the determination of $c_{2}$ (assuming no material depolarization and $100 \%$ absorption on the shutter) due to errors on the determined model parameters.

\begin{tabular}{lcccc}
\hline \hline Parameter & Low value & $\Delta c_{2}$ & High value & $\Delta c_{2}$ \\
\hline Foil potential & 129 & +0.023 & 400 & -0.061 \\
Foil leak & 0.016 & -0.025 & 0.026 & +0.025 \\
$n$ & 0.38 & -0.100 & 2.12 & +0.004 \\
$v_{\text {pk }}$ & 4.37 & +0.007 & 4.73 & -0.002 \\
$v_{\text {cut }}$ & 7.21 & +0.017 & 7.43 & -0.017 \\
DLC potential & 120 & +0.006 & 280 & -0.006 \\
lpb & $0.72 \times 10^{-3}$ & -0.067 & $1.98 \times 10^{-3}$ & +0.027 \\
spec & 0.996 & +0.028 & 1.0 & -0.0002 \\
\hline \hline
\end{tabular}

polarization and $100 \%$ absorption on the shutter. Calculating the transmission with no absorption on the shutter produced no significant effect, which left only the variation of $\bar{\epsilon}$ with depolarization-per-bounce on DLC to be investigated. This was accomplished by varying dpb over a range that incorporated the measurements of Atchison et al. ${ }^{29}$ which found dpb values for DLC between $7 \times 10^{-7}$ and $2 \times 10^{-5}$, consistent with measurements on guides similar to the ones used in this experiment performed by the UCNA collaboration. ${ }^{4,30}$ No significant effect on the average efficiency was observed for variations of $\mathrm{dpb}$ across this range.

The performance obtained here is adequate for the UCNA $\beta$ asymmetry measurement, especially since the experiment currently operates in a bottling mode so that the less efficiently spin flipped (i.e., faster) components of the spectrum have shorter lifetimes in the system, with the lifetime becoming significantly shorter for velocities much greater than $\sim 600 \mathrm{~cm} / \mathrm{s}$, the speed above which the UCN energy exceeds the material potential of the decay trap bottle. ${ }^{9}$ As a result, the equilibrium polarization is dominated by a slower part of the spectrum which is flipped with higher efficiency than the average full-spectrum spin flip efficiency. Not of direct importance to UCNA, then, but nevertheless still of interest, is the fact that the Monte Carlo model predicts a continuing decrease in transmission for increasing spin flip efficiency in contradiction to the power scan results. There is also a discrepancy between the average efficiency calculated by folding Eq. (9) with the longitudinal spectrum at the spin flipper, which gives $\bar{\epsilon}>0.9996$, and the result of the present measurement. These observations are believed to be generally related to characterization of the rf and $B_{0}$ field maps: Since the effect of the shim coil settings used to produce particular gradients was calculated rather than measured in situ, the average $B_{0}$ gradient in the resonance region could differ from expectations. What is more, given the precision with which the $B_{0}$-field profile is known, the existence of multiple resonance points inside the $B_{1}$-field region can not be ruled out. As long as there are an even number of such resonances along all possible trajectories, the spin flip efficiency should approach its theoretical limit for large enough $B_{1}$-field strength, but for an odd number of points satisfying the resonance condition, the spin flip efficiency will be degraded (potentially even decreasing for sufficiently large input power), with the exact scaling dependent on the relative $B_{1}$-field amplitudes and the local gradients at each point satisfying the resonance condition. Of perhaps greater theoretical interest, and not related to precise determination of the fields, is the additional possibility of depolarization associated with bounces in the presence of the rf field. Improvements to the measurement techniques described here, which would involve better characterization of the foil (or simply utilizing an analyzing magnet capable of a larger field) as well as in situ depolarization studies, better characterization of $B_{0}$ and rf field profiles, and transmission measurements at higher powers, should allow the relative contributions of these effects to be resolved and in some cases mitigated. For example, better field maps will allow for more precise optimization of the $B_{0}$ gradient relative to the $\mathrm{rf}$ field profile, which has the potential to enhance the spin flip efficiency of this spin flipper. In addition, since a flipper of 
this type is capable of operating at significantly higher powers than utilized in these tests, minimizing the cavity volume is not critical. This provides the freedom to choose a cavity length, based on the precision of the $B_{0}$-field map and the chosen gradient, which minimizes the possibility of having an odd number of resonance points in the rf field region.

\section{ACKNOWLEDGMENTS}

We are indebted to the wonderful machinists in the NCSU PAMS machine shop as well as to Scott Currie and Henning Back. This work was supported in part by the National Science Foundation (NSF-0700491, NSF-0100689, NSF-0354970, NSF-0653222).

${ }^{1}$ E. Fermi and L. Marshall, Phys. Rev. 71, 915 (1947).

${ }^{2}$ R. Golub, D. Richardson, and S. K. Lamoreaux, Ultra-Cold Neutrons (Adam Hilger, 1991).

${ }^{3}$ A. Steyerl, Z. Phys. 254, 169 (1972).

${ }^{4} \mathrm{M}$. Makela, "Polarized ultracold neutrons: their transport in diamond guides and potential to search for physics beyond the standard model," Ph.D. dissertation (Virginia Polytechnic Institute and State University, 2005).

${ }^{5}$ R. Mammei, APS Meeting Abstracts, BAPS.2009.HAW.KK.7, 2009.

${ }^{6} \mathrm{H}$. Abele, Nucl. Instrum. Methods. Phys. Res. A 440, 499 (2000).

${ }^{7}$ J. M. Pendlebury, Annu. Rev. Nucl. Part. Sci. 43, 687 (1993).

${ }^{8}$ J. Liu, M. P. Mendenhall, and A. T. Holley, et al., Phys. Rev. Lett. 105, 181803 (2010).
${ }^{9}$ A. T. Holley, "Ultracold Neutron Polarimetry in a Measurement of the $\beta$ Asymmetry," Ph.D. dissertation (North Carolina State University, 2012).

${ }^{10}$ A. Abragam, Principles of Nuclear Magnetism (Oxford University Press, 1961).

${ }^{11}$ V. I. Luschikov and Y. V. Taran, Nucl. Instrum. Methods. Phys. Res. 228, 159 (1984).

${ }^{12}$ R. T. Robiscoe, Am. J. Phys. 39, 148 (1971).

${ }^{13}$ A. N. Bazhenov, V. M. Lobashev, A. N. Pirozhkov, and V. N. Slusar, Nucl. Instrum. Methods. Phys. Res. A 332, 534 (1993).

${ }^{14}$ A. Petoukhov, T. Soldner, et al., in Quark-Mixing, CKM-Unitairty (Heidelberg, Germany, 2002), pp. 97-100; e-print arXiv:hep-ph/0312124.

${ }^{15}$ C. E. Hayes, et al., J. Magn. Reson. 63, 622 (1985).

${ }^{16}$ J. Tropp, J. Magn. Reson. 126, 9 (1997).

${ }^{17}$ P. Mansfield, M. McJury, and P. Glover, Meas. Sci. Technol. 1, 1052 (1990).

${ }^{18}$ J. Tropp, J. Magn. Reson. 82, 51 (1989).

${ }^{19}$ S. Crozier, et al., J. Magn. Reson., Ser. B 109, 1 (1995).

${ }^{20}$ S. Crozier, et al., Meas. Sci. Technol. 7, 1083 (1996).

${ }^{21}$ B. J. Dardzinski, S. Li, et al., J. Magn. Reson. 131, 32 (1998).

${ }^{22}$ A. R. Rath, Magn. Reson. Med. 13, 370 (1990).

${ }^{23}$ J. Tropp, J. Magn. Reson. 95, 235 (1991).

${ }^{24}$ R. W. Pattie, et al., Phys. Rev. Lett. 102, 012301 (2009).

${ }^{25}$ A. Saunders, et al., Phys. Lett. B 593, 55 (2004).

${ }^{26} \mathrm{R}$. Mammei, "Thin films for the transport of polarized ultracold neutrons for fundamental symmetry study," Ph.D. dissertation (Virginia Polytechnic Institute and State University, 2010).

${ }^{27}$ C. L. Morris, et al., Nucl. Instrum. Methods Phys. Res. A 599, 248 (2009).

${ }^{28}$ A. T. Holley, APS Meeting Abstracts, BAPS.2007.APR.B16.5, 2007.

${ }^{29}$ F. Atchison, et al., Phys. Rev. C 76, 044001 (2007).

${ }^{30}$ R. Rios, APS Meeting Abstracts, BAPS.2009.APR.C14.3, 2009. 